\title{
Influencing Climate Change Attitudes in the United States: A Systematic Review and Meta-Analysis
}

\author{
Jacob B. Rode, ${ }^{1}$ Amy L. Dent, Caitlin N. Benedict, Daniel B. Brosnahan, Ramona L. Martinez, ${ }^{2}$ \\ and Peter H. Ditto \\ Department of Psychological Science, University of California, Irvine, \\ 4201 Social and Behavioral Sciences Gateway, Irvine, CA 92697-7085
}

\section{Author Note}

Jacob B. Rode (iD) https://orcid.org/0000-0001-8289-7760

All data and study materials are publicly available at: https://osf.io/q7jhe/. Declarations of interest: none. This research did not receive any specific grant from funding agencies in the public, commercial, or not-for-profit sectors.

Correspondence concerning this article should be addressed to Jacob Rode at jbenjaminrode@gmail.com

This is a post-peer review, pre-copyedit version of an article published in the Journal of Environmental Psychology. The published version can be found at:

\section{https://doi.org/10.1016/j.jenvp.2021.101623}

\footnotetext{
${ }^{1}$ Jacob B. Rode is now at Harvard University

${ }^{2}$ Ramona L. Martinez is now at the University of California, Riverside
} 


\section{Acknowledgements}

We thank Paul Piff, Joanne Zinger, and Steven Davis for their insightful feedback at various stages of this project. We also thank the primary study authors who were willing to share their data with us. We thank anonymous reviewers for their help with the manuscript, particularly with contextualizing the size of the summary effect. 


\begin{abstract}
Researchers interested in climate change communication have investigated how people respond to messages about it. Through meta-analysis, the current research synthesizes the multitude of experimental studies on this topic to uncover which interventions are most effective at influencing attitudes about climate change. The meta-analysis focuses on experimental studies that included a control condition and measured climate change attitudes among participants in the United States. After a large literature search, 396 effect sizes were retrieved from 76 independent experiments ( $N=76,033$ participants). Intervention had a small, significant positive effect on attitudes, $g=0.08,95 \%$ CI $[0.05,0.10], 95 \%$ prediction interval $[-0.04,0.19], p<.001$. Surprisingly, type of intervention was not a statistically significant moderator of this effect, nor was political affiliation. However, type of attitude was a significant moderator: the treatmentcontrol difference in attitudes was smaller for policy support than for belief in climate change, indicating that policy attitudes are more resistant to influence than belief in climate change. Interventions that aimed to induce skepticism (e.g., misinformation) had a significantly stronger average effect on attitudes than did ones that intended to promote belief in climate change, suggesting that belief in climate change is more easily weakened than strengthened.

Keywords: climate change, attitudes, meta-analysis, interventions
\end{abstract}

Word count: 11,003 (excluding references, footnotes, and the Appendix) 


\section{Influencing Climate Change Attitudes in the United States:}

\section{A Systematic Review and Meta-Analysis}

\section{Introduction}

Although immediate action can substantially reduce the negative consequences of climate change (IPCC, 2018), public beliefs and willingness to act do not reflect accurately the scientific evidence on climate change and emissions reductions needed to mitigate it. Specifically, whereas $97 \%$ of climate scientists agree that human-caused global warming is occurring, a recent poll found that only $62 \%$ of the American sample reported that they believe global warming is human-caused (Leiserowitz, Maibach, Rosenthal, Kotcher, Bergquist, et al., 2020). Additionally, support for action on climate change is fractured along political lines. For example, $83 \%$ of Democrats, $56 \%$ of Independents, and $22 \%$ of Republicans reported that global warming should be a high priority for the President and Congress (Leiserowitz, Maibach, Rosenthal, Kotcher, Ballew, et al., 2020). Taken together, these polls reveal that Americans are divided politically -with Independent and Republican attitudes further away from scientific consensus than Democrat attitudes -- and they differ in beliefs about the cause of global warming, its potential effects, policies that should be enacted to curtail them, and its priority as a voting issue (Leiserowitz, Maibach, Rosenthal, Kotcher, Ballew, et al., 2020). Therefore, social scientists have investigated ways of bridging the gap between public and scientific knowledge about climate change, as well as examining ways of inducing support for policies to mitigate it.

\subsection{Interventions to Influence Climate Change Attitudes}

When considering how to alter perceptions about climate change, researchers have proposed a number of interventions, including making climate change more concrete and local, tailoring messages to the audience's values, and using messages to assuage defensive reactions 
(Fielding et al., 2014; Weber, 2016). While previous reviews have discussed interventions (e.g., Markowitz \& Shariff, 2012; Pearson et al., 2016; Weber, 2016), a comprehensive quantitative synthesis has not yet been conducted. Combining studies quantitatively can help determine which interventions are effective at influencing climate change attitudes and for whom. A recent meta-analysis more narrowly tested the effect of message frames on various climate change attitudes and behavioral intentions, analyzing 27 effects sizes from nine published studies (Li \& $\mathrm{Su}, 2018)$. The current meta-analysis expands the scope of interventions to include any type of experimental manipulation across a larger range of studies. In particular, the current metaanalysis integrates and reconciles experimental studies of interventions designed to influence climate change attitudes for people in the United States while identifying potential moderators of their effectiveness. We turn to those moderators next.

\subsection{Moderators of Intervention Effectiveness}

\subsubsection{Political Affiliation}

Political ideology is a strong predictor of climate change beliefs, and there is evidence that political party in particular drives differences in support for attempts to address climate change (Ehret et al., 2017; Hornsey et al., 2016; McCright \& Dunlap, 2011). Researchers have examined strategies to communicate climate change in a way that increases acceptance of it or endorsement of policies, especially among conservatives and those most skeptical of climate change. Therefore, it is important to test the differential effectiveness of interventions among liberals, conservatives, and moderates.

Research on motivated reasoning provides some predictions about how individuals may process information about climate change. Motivated reasoning suggests that individuals have a desire to reach certain conclusions when forming attitudes and beliefs. Specifically, individuals 
can have directional goals (i.e., reach a preferred conclusion) or accuracy goals (i.e., reach an accurate conclusion) and will employ strategies consistent with those goals (Kunda, 1990). In addition, motivated reasoning can take place in the selection of information (e.g., news sources) and in the processing of information (i.e., how one's beliefs change in response to new information). Research on motivated reasoning tends to focus on directional goals, emphasizing situations where individuals selectively expose themselves to preferred information (Garrett, 2009; W. Hart et al., 2009; Stroud, 2010) or are too easily accepting of preferred information (Ditto et al., 2019; Ditto \& Lopez, 1992; Taber \& Lodge, 2006). Within the realm of climate change, there is some evidence of directional motivated reasoning (P. S. Hart \& Nisbet, 2012; Kahan et al., 2017; Palm et al., 2017). Other research, however, finds little evidence of directional motivated reasoning in climate change communication, suggesting that perhaps people may rely on accuracy goals when interpreting climate change information (Druckman $\&$ McGrath, 2019; van der Linden, Maibach, et al., 2019). Traditional research on motivated reasoning may be "too hot", assuming more directional, political motivated reasoning than the evidence suggests (Hennes et al., 2020).

A recent meta-analysis highlights evidence that political partisans tend to reject information that is counter to their pre-existing beliefs (Ditto et al., 2019), suggesting that conservatives will be less influenced by climate change interventions (i.e., smaller effect sizes) than liberals. If conservatives are motivated to form accurate conclusions, then some interventions might be effective like those that provide evidence perceived as strong by conservatives (Druckman \& McGrath, 2019). However, it is unlikely that many of the traditional science-based interventions will consist of evidence perceived as strong by conservatives, given their distrust of climate science (Lewandowsky \& Oberauer, 2016; Washburn \& Skitka, 2018). If 
conservatives are motivated to form directional conclusions, then interventions may be effective if they affirm conservative values (Druckman \& McGrath, 2019). Given the specific nature of these two elements -- interventions that provide strong evidence as perceived by conservatives or affirm conservative values -- it seems unlikely that many interventions will fit either situation. Therefore, it is expected that most interventions are interpreted in a partisan manner, leading to rejection of the information rather than change in attitudes and thus smaller effect sizes for conservatives.

Relatedly, there is a line of research that investigates how attitude measures are phrased and whether they use the term "global warming" or "climate change." Some evidence suggests that the phrasing is important for conservatives (Schuldt et al., 2011), such that conservatives are more likely to believe in climate change than in global warming. Other studies show that Independents are especially influenced by the phrase choice (Benjamin et al., 2017). As this line of research compares "global warming" to "climate change" (rather than treatment vs. control), it is unclear whether interventions using one phrase or the other would be more effective and therefore we do not propose a hypothesis.

\subsubsection{Type of Attitude}

A second theoretical moderator is the type of climate change attitude. Attitudes can include belief that climate change is happening, belief that climate change is caused by humans, concern about climate change, and support for climate change mitigation policy, among many others. Public polling reveals differences across these attitudes. For example, a recent poll found that around $73 \%$ of Americans believe in global warming, $62 \%$ believe it is human-caused, $66 \%$ are at least somewhat worried about it, and $44 \%$ say they have experienced its effects (Leiserowitz, Maibach, Rosenthal, Kotcher, Bergquist, et al., 2020). While support for climate 
change policy varies depending on the specific policy, Hornsey et al. (2016) provided metaanalytic evidence that the link between climate change belief and policy support is weak, suggesting distance between these two attitudes. One reason for this distance may be that any climate change policy would likely require a cost (e.g., tax), making it more difficult to garner support for policy than belief. Research finds that policies framed as taxes are especially disliked, highlighting that cost may play a role in policy support (Hardisty et al., 2010, 2019). This could make it even more difficult for interventions to increase it.

In addition, there is evidence that liberals and conservatives respond differently across these attitude outcomes. As public opinion polling has found, liberals have a strong belief in anthropogenic climate change, whereas many conservatives believe that the earth is warming but not due to human activity (Leiserowitz, Maibach, Rosenthal, Kotcher, Ballew, et al., 2020). Additionally, research suggests that support for climate change policy could be diminished among conservatives due to the regulatory nature of policy implementation (Campbell \& Kay, 2014). This finding indicates that liberal and conservative differences in policy support might be larger than differences in climate change belief.

However, other research suggests the opposite conclusion. Renewable energy legislation can garner conservative support (Gillis \& Popovich, 2017), especially when it is framed in line with conservative ideology (Hess et al., 2016). Recent polling found that Trump voters support policies such as generating renewable energy on public land (77\%), regulating pollutants that cause global warming (62\%), and funding more research in clean energy (71\%; Leiserowitz et al., 2017). Corroborating these results, a recent media article noted that Trump won many of the country's top wind energy producing states in the most recent election (Gillis \& Popovich, 2017). Therefore, although there seems to be more evidence to suggest that liberals and 
conservatives respond differently to policies about climate change, other data suggests liberal and conservative attitudes towards policy may be closer than they are for other types of attitudes. Importantly, given that the current meta-analysis is focused on climate change, there may be less conservative support for the policies included in this meta-analysis than for other general environmental policies such as recycling policies.

\subsubsection{Type of Intervention}

Key to the current meta-analysis is an examination of which types of interventions are most effective. This section will provide a brief background about various intervention types.

\subsubsection{General or Scientific Information About the Effects of Climate Change.}

Common interventions involve describing the effects of climate change, whether through introducing a new scientific finding or simply providing general information. Within the field of climate change communication, the information-deficit model posits that increasing public knowledge will lead to increased public support to address climate change (for a review, see Suldovsky, 2017). Some evidence suggests that knowledge about climate change may be beneficial for generating action on global warming and correcting misperceptions (Bord et al., 2000; Guy et al., 2014; Ranney \& Clark, 2016).

Responses to general information about climate change, however, may not be evenly distributed across the political spectrum. Some researchers and journalists argue that conservatives are more likely to deny science or have less trust in it (Gauchat, 2012; Jost et al., 2003; Mooney, 2012). However, a recent experimental study revealed that both liberals and conservatives engage in denying scientific information that is not in accordance with their attitudes (Washburn \& Skitka, 2018). Conservatives are less trusting of climate science than 
liberals are (e.g., Pechar et al., 2018), suggesting that additional information about climate change may be persuasive for liberals but not for conservatives.

1.2.3.2 Scientific Consensus. A related line of research has emerged in the past few years focusing on messaging about agreement among climate scientists. This research attempts to bridge the gap between science and the public. Often based on the gateway belief model (GBM), research on this topic posits that knowledge of the scientific consensus is a gateway belief to other climate change attitudes, such as belief in human-caused climate change and support for action to address it (van der Linden, Leiserowitz, et al., 2019; van der Linden et al., 2015).

Multiple experimental studies have examined the effectiveness of consensus messaging, with many providing support for the GBM (e.g., Brewer \& McKnight, 2017; Myers et al., 2015; van der Linden, Leiserowitz, et al., 2019; van der Linden et al., 2015). However, the overall effectiveness of consensus messaging -- especially when looking at attitudes such as belief and support for policy -- is debated (Dixon et al., 2017, 2019; Kahan, 2017; Kerr \& Wilson, 2018; Ma et al., 2019; van der Linden et al., 2017, 2018; van der Linden, Maibach, et al., 2019). Experimental evidence on consensus messaging is mixed regarding whether effective consensus messages merely increase participants' beliefs about the scientific consensus itself or if they also increase other climate change attitudes, such as belief and policy support.

1.2.3.3 Emotion. Because of the potential catastrophic effects of climate change, discussion of it often creates anxiety and fear. Media members debate whether appeals to fear are better at compelling action than optimistic calls for hope (Mann et al., 2017; Wallace-Wells, 2017). Meta-analyses on fear appeals suggest that it may be an effective motivator depending on other aspects of the message (Tannenbaum et al., 2015; Witte \& Allen, 2000). Although fear- 
based messaging in other domains is somewhat effective, its success within the environmental domain is mixed (see Reser and Bradley, 2017, for a review). Researchers have also examined the link between positive emotions and climate change attitudes (Smith \& Leiserowitz, 2014). However, Chapman et al. (2017) criticized the use of emotional messages as an effective tool in climate change communication, citing mixed evidence of emotions research and the unpredictable ways in which people respond to emotional messages. Given the range of responses they can provoke, the effectiveness of emotional messages relies on the combination of the emotion used to influence attitudes and the audience receiving the message. ${ }^{1}$

1.2.3.4 Psychological Distance. Because climate change is an abstract and difficult to grasp concept, it can be challenging for people to feel urgency in addressing it (e.g., Markowitz \& Shariff, 2012; Moser, 2010). Researchers have proposed that one way to increase engagement with climate change is to make it feel closer and less abstract (Fielding et al., 2014; Weber, 2016). Relying on construal level theory (CLT; Trope \& Liberman, 2010), research using psychological distance manipulations attempts to make climate change feel closer psychologically. Some correlational studies suggest that people have stronger attitudes toward climate change the closer they feel to it (A. S. Singh et al., 2017). Although theoretically expected to increase climate change attitudes, the experimental evidence for the effectiveness of psychological distance interventions is mixed (McDonald et al., 2015), perhaps because reduced distance may increase fear (Spence \& Pidgeon, 2010) or because CLT may not be a good theoretical fit for climate change (Brügger, 2020). This mixed evidence leaves doubt as to whether these interventions will be effective overall.

\footnotetext{
${ }^{1}$ Although there were not enough emotion-related studies to group them by emotion (e.g., fear, hope) in the current meta-analysis, future meta-analyses on the topic should continue to consider the differential effect of emotions on climate change attitudes and other outcomes.
} 
1.2.3.5 National Security. National security interventions highlight the benefits of addressing climate change through the lens of national security (e.g., increased energy independence), which may be more relevant for conservatives than for liberals. In a metaanalysis of political ideology and personality, Jost et al. (2003) found that conservatism was associated with death anxiety, system instability, uncertainty tolerance, and fear of threat and loss -- all constructs related to security. However, liberals and conservatives in the United States share a common ingroup identity as Americans so have a vested interest in its success, which makes an appeal to national security a potentially effective intervention for both groups (e.g., Wolsko, 2017).

1.2.3.6 Economy. Economic interventions highlight the economic benefits of addressing climate change or the economic harms of failing to do so. These interventions rely on Americans' (and particularly conservatives') widely-held desire for a healthy economy (Pew, 2016). Conservatives may be particularly attuned to economic issues as conservatism as an ideology generally favors small government and a free market economy (e.g., Crowson, 2009; Everett, 2013). Therefore, a message that emphasizes the economic aspects of climate change impacts -- whether economic benefits from mitigation or economic costs of impacts -- speaks a conservative language by highlighting values often associated with conservatism. ${ }^{2}$

1.2.3.7 Religion. Religious interventions included in the current meta-analysis generally take on two forms: arguing for environmental action by relying on religious values or texts and highlighting a religious figure's (e.g., Pope Francis) support for environmental causes. These

\footnotetext{
2 'Economy' may have some nuance as a category. Generally, conservatives support the free market whereas liberals favor government regulation. An intervention that highlights the ability of the free market to stop climate change is slightly different than one that highlights the harm that climate change will have on the economy (or positive economic benefits of mitigation). Unfortunately, the small number of economic manipulations involved in the current meta-analysis limits our ability to distinguish between types of economic interventions.
} 
interventions leverage religion by linking the environment to Christian values in order to target Christian participants' morality in hopes that this might be especially persuasive for climate skeptics who are also Christian. ${ }^{3}$ Given this targeted nature of religious interventions, however, it is unlikely that they would be effective across multiple studies with religiously diverse samples.

1.2.3.8 Morality. Given that moralized attitudes (those rooted in one's sense of moral right or wrong) are fundamentally different from non-moralized attitudes (Skitka et al., 2005), research using moral interventions attempts to increase engagement with climate change by reframing it as a moral issue. Some studies suggest that aligning a climate message with the moral values of its audience is an effective way to break through political polarization. Specifically, reframing climate change in terms of conservative morality (see Graham et al., $2009,2011)$ seems to be an effective way to engage conservatives in climate change (Feinberg \& Willer, 2013; Wolsko, 2017; Wolsko et al., 2016). Other studies have used less specific moral messaging as an intervention to moralize climate change, relying on moral intuitions to drive support for action on climate change (Markowitz \& Shariff, 2012).

1.2.3.9 Summary. While the evidence for each intervention type is complex, interventions that target specific audiences seem most promising. In particular, moral, economic, and national security interventions include studies that show strong positive effects for conservatives. The effectiveness of more general interventions, such as those that include information about the effects of climate change, is less clear due to the range of evidence for and against them.

\subsubsection{Type of Sample}

\footnotetext{
${ }^{3}$ Most research on religion and climate change (and all of the studies included in the current meta-analysis) focuses on Christianity specifically.
} 
The type of sample that studies recruit may play a role in the effectiveness of their interventions. Many studies within the field of climate change communication recruit college students, Amazon Mechanical Turk (MTurk) workers, or a nationally representative sample. In comparing MTurk and nationally representative samples, one concern is that participants recruited through MTurk are more politically liberal (Berinsky et al., 2012; Levay et al., 2016). There is some evidence that the conservatives on MTurk are similar to conservatives in general, whereas the liberals are slightly more liberal (Clifford et al., 2015). This asymmetry in strength of political identity implies interventions that are particularly effective for liberals are also more effective for MTurk samples than for nationally representative samples. However, research generally finds that MTurk samples provide quality data and perform similarly to nationally representative samples in experiments (Buhrmester et al., 2011; Casler et al., 2013; Crump et al., 2013; Kees et al., 2017).

Historically, college student samples have not been representative of the general public in psychology studies (Sears, 1986). Some polling evidence suggests that young Republicans and young people in general are more supportive of action to curb emissions than the general population (Mooney et al., 2014). Another recent poll shows that $77 \%$ of American adults under 30 years-old say the environment should be a top governmental priority and $64 \%$ say climate change should be (Pew, 2020). These numbers reflect a 10\% and $8 \%$ increase over adults aged 30 to 49 for the environment and climate change, respectively. This generational shift implies that college student conservatives might be less skeptical of climate change than the average American conservative. As a result, there may potentially be a ceiling effect for college students, such that interventions are not effective simply because college students already have extremely strong positive attitudes towards climate change. Indeed, if samples vary on important 
moderators of an effect (e.g., ideology), they may be prone to display differential treatment effects (Boas et al., 2020; Druckman \& Kam, 2011).

\subsection{The Current Meta-Analysis}

In the previous section, we discussed reasoning for why intervention effectiveness might differ based on political affiliation, type of attitude, type of intervention, and type of sample. The current meta-analysis tests if interventions work overall then various moderators to identify factors of theoretical, practical, and methodological importance that explain variation in effect sizes across studies. Because politics is a key driver of climate change attitudes, we test each moderator individually then its interaction with political affiliation. This meta-analysis provides insight into factors that lead to successful interventions with the goal of helping identify the best ways to spur collective action on climate change.

\section{Materials and Methods}

\subsection{Inclusion Criteria}

Studies had to meet the following three criteria to be included in the meta-analysis: 1) random assignment of participants into study conditions, 2) a control condition where participants did not receive an intervention related to climate change, and 3) a measure of climate change attitudes after the experimental manipulation. Studies qualified for inclusion regardless of their publication status and were not excluded based on publication type, publication year, or publication quality.

\subsection{Exclusion Criteria}

Given our focus on climate change attitudes and American ideological polarization about it, studies were excluded if their samples were comprised of participants outside the United States (with some empiricl evidence pointing to the unique nature of climate change skepticism 
in America; Hornsey et al., 2018). Studies were also excluded if they only measured general environmental attitudes or attitudes toward environmental issues not directly related to climate change (e.g., recycling). Given our focus on climate change attitudes, we limited the population of studies to those that measured attitudes rather than behaviors or behavioral intentions since other meta-analyses have studied pro-environmental behavior (e.g., Maki et al., 2016, 2019), household energy use (Karlin et al., 2015), the relation between climate change attitudes and behavior (Hornsey et al., 2016), and behavioral interventions for household action on climate change (Nisa et al., 2019).

\section{Figure 1}

Flow Chart of Abstract and Full-Text Screening Process

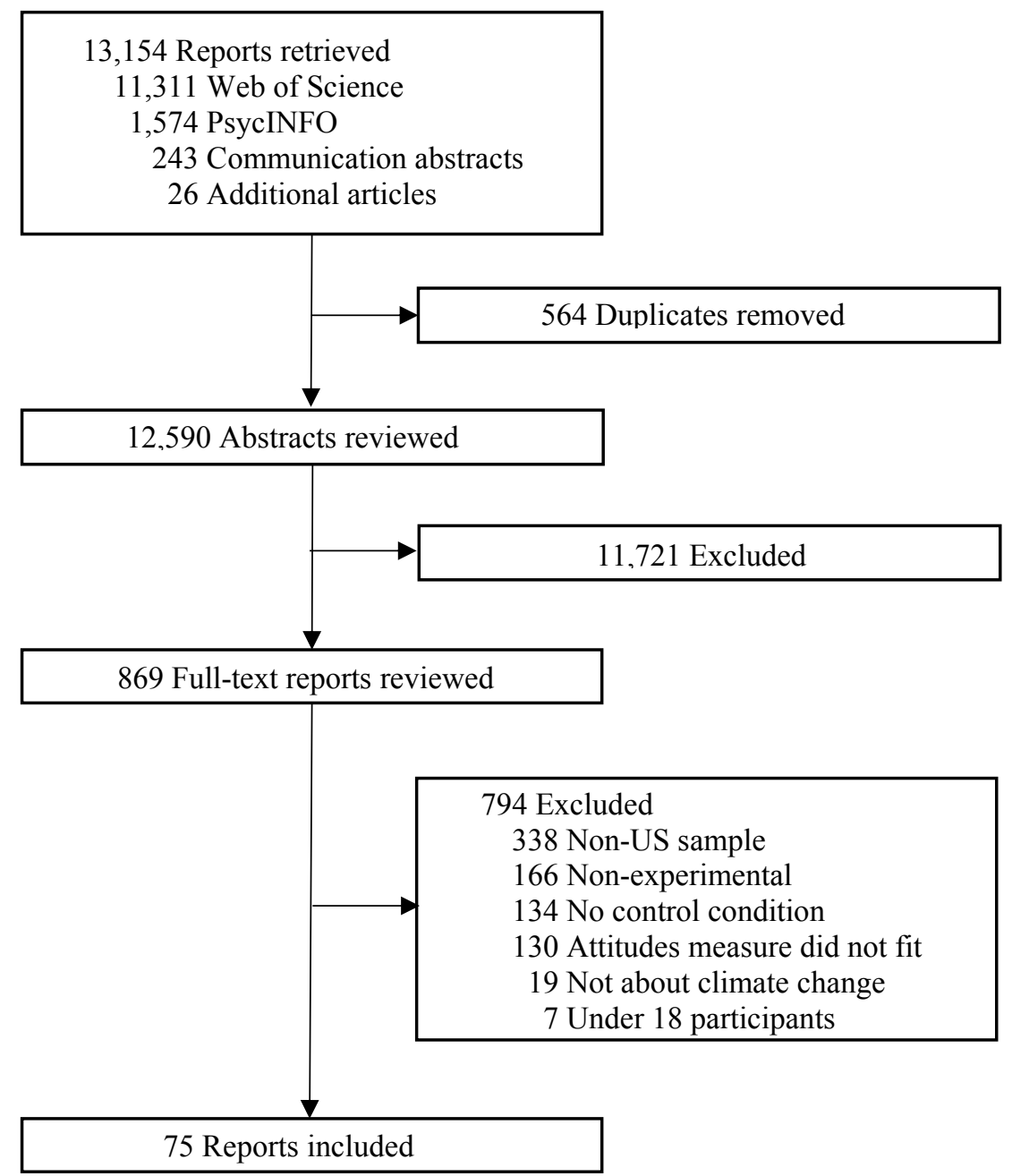




\subsection{Searching the Literature}

Three complementary search strategies were adopted to collect research relevant to our meta-analysis based on these inclusion and exclusion criteria. First, an electronic database search was conducted using Web of Science, PsycINFO, and Communication Abstracts between early February and mid November of 2019. In early 2020, the electronic database search was updated. As displayed in Table 1, the search terms reflected three aspects of our meta-analysis parameters: measuring attitudes, using an experimental design, and investigating the topic of climate change. Second, direct contact strategies were adopted to complement the electronic database search and offset its bias toward published studies. In particular, we emailed six of the authors with multiple studies qualifying for the meta-analysis to inquire if they had or knew of unpublished data on this topic. We also sent an email to the Society for Personality and Social Psychology listserv with a request for unpublished data. Third, we scanned the references of each study that qualified for inclusion. Figure 1 displays these literature search strategies.

\subsection{Screening the Literature}

The first author conducted both abstract and full-text screening of reports retrieved through these literature search strategies (the abstract screening tool can be found at: https://osf.io/q7jhe/). The first author screened the full-text of research reports for our inclusion and exclusion criteria if all abstract screening criteria were met or the report was ambiguous about any of these criteria and for any report without an abstract.

\subsection{Coding Studies}


Studies were coded for general characteristics about the report (e.g., type of publication), sample (e.g., proportion female, sample location), intervention (e.g., type and description), attitude (e.g., type), and effect size (full coding protocol at: https://osf.io/q7jhe/). Each report was coded by the first author and one other trained researcher (e.g., research assistant or graduate student). Coding agreement was calculated for the coding of each moderator and effect size with either unweighted Cohen's kappa or the intra-class correlation coefficient (ICC) as appropriate (values reported throughout). Discrepancies were then resolved through discussion, therefore ultimately dropping the percentage of disagreement to zero afterward.

\section{Table 1}

Summary of Electronic Database Searches

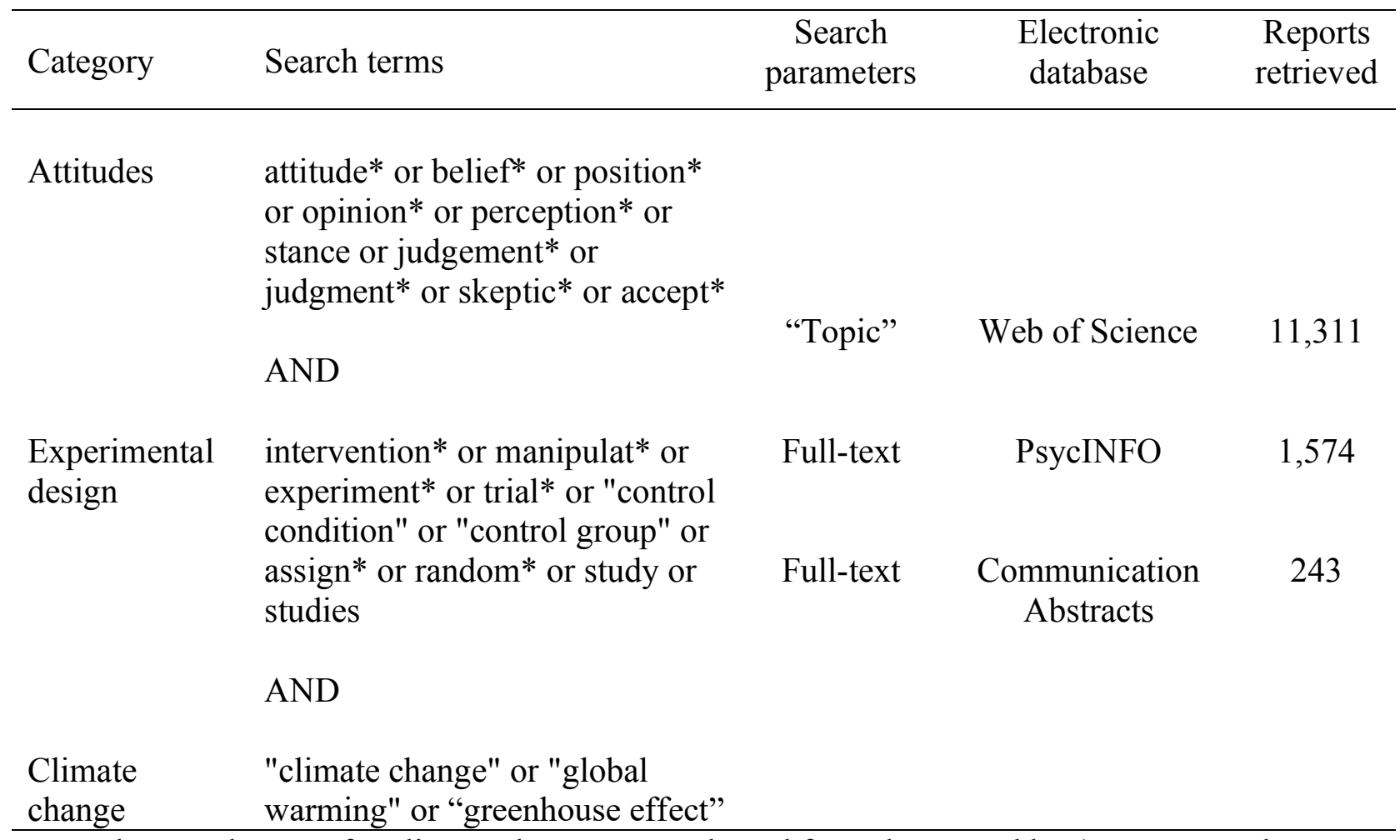

Note. The search terms for climate change were adapted from those used by (Hornsey et al., 2016). 


\subsection{Moderators}

\subsubsection{Political Affiliation}

For every study, effect sizes were calculated separately for the full sample, liberals/Democrats, conservatives/Republicans, and moderates/Independents. Studies tended to include a measure of political party (i.e., Democrat, Republican, Independent), political ideology (i.e., liberal, conservative, moderate), or both. If both were measured, we requested data for and calculated effect sizes from the measure of political party, due to its strength of association with climate change belief (Hornsey et al., 2016). Otherwise, we requested and used the political measure that was available in the report (see the Supplemental Materials for more information on the non-typical measures of political affiliation).

\subsubsection{Type of Attitude}

Before coding, we specified eight potential categories, including an "other" option (see coding protocol). After coding studies, we combined some of the original categories and analyzed type of attitude with these updated six categories: belief that climate change is happening (19.5\% of effect sizes), belief in human-caused climate change (19.5\%), concern or worry about climate change $(9.8 \%)$, risk of climate change $(13.8 \%)$, support for climate change policy $(23.6 \%)$, and other $(13.8 \%) .{ }^{4}$ Coding agreement calculated with the original coding categories $(k=.78)$ and the updated categories $(k=.88)$ showed substantial and nearly perfect agreement, respectively (Landis \& Koch, 1977).

2.6.2.1 Phrasing: Climate Change or Global Warming. In addition to coding the type of attitude, we also coded whether it was phrased in terms of climate change, global warming,

\footnotetext{
${ }^{4}$ We also analyzed this variable using the original categories and the pattern of results was consistent. The Supplemental Materials include more details on the combining of categories.
} 
both, or neither. In moderator analyses, we only compared the effect sizes coded as global warming and climate change $(k=.79)$ and tested their interaction with political affiliation.

\subsubsection{Type of Intervention}

In the coding protocol, we outlined seven categories that we anticipated finding in the included studies: morality, emotion, general or scientific information about the effects of climate change, scientific consensus, psychological distance (near), psychological distance (far), and other. Because many interventions included manipulations that fit into multiple categories (e.g., the intervention included effects of climate change and emphasized how they are occurring nearby or far away), coders selected all categories that applied to an intervention. This classification mostly led to mutually exclusive categories among the six expected intervention types (i.e., excluding the "other" category). ${ }^{5}$ One exception was particularly problematic, specifically information about the effects of climate change: while $34 \%$ of interventions included information about the effects of climate change, only $9 \%$ exclusively contained this type.

Therefore, we categorized interventions as information about the effects of climate change if that was listed as the only category. Additionally, a number of interventions in the "other" category coalesced into themes: national security, religion, and economy/free market (smaller categories also emerged, but with very few studies). Moderator tests were conducted on the original coding of intervention type and the expanded types (with the three new categories). Both the original coding of the intervention type $(k=.76)$ and the expanded types $(k=.74)$ indicated substantial agreement between coders.

2.6.3.1 Intervention Direction. Although not originally planned as a moderator, intervention direction emerged as an important classification. Some studies included conditions

\footnotetext{
${ }^{5}$ The original coding and the expanded coding had a few overlaps between type of interventions. Full details about those overlaps are described in the Supplemental Materials.
} 
that were not interventions to increase climate change attitudes but rather to introduce misinformation or anti-climate change arguments. Many of these interventions included misinformation as part of a manipulation, usually to compare it with a corresponding correction condition (i.e., a condition that includes both misinformation and debunking of misinformation). To avoid confounding effect size estimates by combining data from interventions designed to increase belief in climate change with those intended to decrease it, we coded whether interventions were positive (i.e., presenting information to increase belief in climate change), mixed (i.e., presenting information to increase belief in climate change along with a skeptic's viewpoint or counterarguments), or negative (i.e., presenting only a skeptic's viewpoint or information to decrease belief in climate change). Manipulations that humorously presented a skeptic's viewpoint as a way to reveal the weakness of that argument were coded as positive (e.g., Anderson \& Becker, 2018). Intervention direction was treated as a categorical variable with positive interventions as the reference group (coded 0) and dummy variables to represent mixed and negative interventions (coded 1 for each vector). There was substantial agreement between coders on intervention direction $(k=.63)$.

2.6.3.2 Intervention Medium. Another moderator that emerged was the type of medium through which interventions were delivered, including short text excerpts, videos, and images or maps of climate change effects. We coded this moderator into five categories: text, video, image or map, text along with image or map, and other. These initial categories were then combined into four: text, video, image or map, and other $(k=.80){ }^{6}$

2.6.3.3 Control Type. As a methodological moderator, we coded the type of control condition within each experiment. Coders sorted control conditions into five categories (see

\footnotetext{
${ }^{6}$ Studies with text accompanied by images or maps were grouped into the image or map category. One study included both text and a table, which was grouped into the text category.
} 
coding protocol), ranging from no information at all to a brief definition of climate change.

Because most interventions used either no information or an unrelated task, we grouped this moderator into three categories: no intervention, a task unrelated to the environment or climate change, and other $(k=.88)$.

\subsubsection{Type of Sample}

Coders selected from six options to categorize samples: college students, MTurk, YouGov, Qualtrics Panel, GfK, and other. Most studies used MTurk (23 samples, 30\%), college students (14 samples, 18\%), Survey Sampling International (SSI; 9 samples, 12\%), and Qualtrics Panels (6 samples, $8 \%$ ). The remainder of sample types were widely distributed and none were used by more than three independent experiments. Additionally, we categorized whether samples were nationally representative and used this distinction as a separate moderator. Coders showed nearly perfect agreement for both sample type $(k=.98)$ and whether the sample was nationally representative $(k=.81)$.

\subsubsection{Sample and Report Characteristics}

To examine demographic variables as moderators, we coded the average age (or median when mean was not available) for each experiment $(k=.81),{ }^{7}$ the percent of the sample that was female $(k=.99)$, and the percent of the sample that was White $(k=.94)$. Additionally, coders noted the year that the data were collected (if not available, then the year the manuscript was submitted) and the type of publication (e.g., journal article, dissertation, unpublished study). Publication type was grouped as published or unpublished and tested as a moderator.

\subsection{Effect Size Calculation}

\subsubsection{Standardized Difference Between Means (SMD)}

\footnotetext{
${ }^{7}$ After removing a typo (where .478 was changed to 47.8 for average age), the kappa for age increased to nearly perfect agreement $(k>.99)$.
} 
Given that Cohen's $d$ tends to overestimate the population mean difference in small samples (Borenstein et al., 2009), Hedges' $g$ is seen as preferable when working with small samples (Lakens, 2013). Because we split effect sizes by political affiliation (i.e., an effect size for each political group), the samples tended to be small. Therefore, we calculated Cohen's $d$ for the standardized difference between means (treatment vs. control) and applied the correction for small samples used by Hedges' $g$ (Hedges, 1981).

The first author calculated all effect sizes using the effect size calculator from the Campbell Collaboration (Wilson, n.d.), and all effect sizes were also coded by another trained researcher (ICC for $d=.85$; ICC for $v=.91$ ). Discrepancies (where differences in $d$ were greater than 0.01 and for $v$ greater than 0.001 ) were then resolved by discussion. All effect sizes were calculated such that larger effects corresponded to stronger climate change attitudes in the intervention group compared to control (e.g., more belief, more concern, more worry, more policy support). If studies included attitudes in the opposite direction (e.g., climate change skepticism), we flipped the sign of its calculated effect size.

\subsection{Meta-Analytical Approach}

\subsubsection{Dependent Effect Sizes}

Because many experiments contained multiple interventions (dependent effect sizes from a shared control condition) and multiple types of attitudes (dependent effect sizes from the same participants completing multiple attitude measures), we used robust variance estimation (RVE), which is a random-effects meta-regression technique that accounts for dependent effect sizes within each study (Hedges et al., 2010). RVE does not make distributional assumptions about effect sizes (Hedges et al., 2010), provides valid estimates about meta-regression coefficients even in small samples (with a small sample adjustment, Hedges et al., 2010; Tipton, 2015), and 
includes meta-regression moderator tests (Tipton \& Pustejovsky, 2015). Relying on the robumeta package (Fisher \& Tipton, 2015) in R, we used RVE in the current meta-analysis because it allowed us to utilize the maximum amount of information from the included studies (e.g., using all attitude measures). We would not expect interventions to produce a single true effect size, with a random-effects model (as opposed to fixed) appropriate as a result.

\subsubsection{Weighting}

In RVE, weights are determined by the number of effect sizes per study, the average variance (across effect sizes) in a study, and the estimate of the between-study variability, Tausquared $\left(\tau^{2}\right)$. Because most of the dependencies in our meta-analytic dataset were due to multiple measures and a shared control group, we used a correlated effects model along with the corresponding weighting scheme (Hedges et al., 2010; Tanner-Smith \& Tipton, 2014).

\subsubsection{Moderator Analyses}

We used the Approximate Hotelling-Zhang (AHZ) test for moderator analysis (TannerSmith et al., 2016; Tipton \& Pustejovsky, 2015), implemented with the clubSandwich package (Pustejovsky, 2020) in R. In RVE, $T^{2}$ represents an estimate of the variance between-studies after averaging dependent effect sizes within-study. The calculation of $T^{2}$ relies in part on the value of a common correlation. The most conservative estimate of this correlation is 1 , which would mean that studies do not receive extra weight for having more effect sizes (Fisher \& Tipton, 2015; Hedges et al., 2010). We used the default assumed correlation of .80, but sensitivity tested correlations of 0 and 1 and report whenever the pattern of results changes among these assumptions (although results tend to be robust to this correlation assumption; Hedges et al., 2010; Tipton, 2015).

\section{Results}




\subsection{Retrieving Effect Sizes}

75 reports qualified for inclusion in the meta-analysis (Figure 1). ${ }^{8}$ The meta-analysis ultimately integrated 63 reports with 76 independent samples and 396 effect sizes from 76,033 participants. For each experiment, we calculated an effect size for the full sample as well as an effect size for liberals, conservatives, and moderates separately. Where political affiliation is not included as a moderator in the results reported below, the meta-analytic calculations are based on effect sizes from the full samples.

\subsection{Summary Effect}

Combining all studies led to a very small effect and a large amount of heterogeneity, $g=$ $0.03,95 \%$ CI $[-0.01,0.06], p=.19, I^{2}=80.47$, as expected due to the varied nature of interventions and other factors that were hypothesized to impact their effect. We first tested moderation by intervention direction in order to exclude interventions that were intentionally aiming to reduce belief in climate change (e.g., providing misinformation about climate change). As expected, intervention direction was a significant moderator, $F(14.41)=15.88, p<.001$ (Figure 2). In particular, interventions aiming to reduce climate change belief were significantly different than interventions aiming to increase it, $b=-0.39,95 \% \mathrm{CI}[-0.53,-0.24], t(11.46)=-$ $5.82, p<.001$. Interventions that combined these elements were not significantly different than positive interventions, $b=-0.06,95 \% \mathrm{CI}[-0.13,0.002], t(11.73)=-2.13, p=.06$. Separately meta-analyzing these interventions results in negative interventions having the largest effect size $(g=-0.29)$, followed by positive $(g=0.08)$ and mixed interventions $(g=0.01)$.

\footnotetext{
${ }^{8}$ Out of these 75 reports, 15 reported the information needed to calculate effect sizes. We emailed the corresponding authors of the remaining 60 reports and received 48 responses that included the necessary data to calculate effect sizes, resulting in a final sample of 63 reports.
} 
This evaluation of intervention direction reflects the theoretical reasoning behind separating positive, mixed, and negative interventions. For the remaining analyses, we excluded negative (27 effect sizes from 14 studies) and mixed (57 effect sizes from 12 studies) interventions to isolate the effect of positive interventions on climate change attitudes. ${ }^{9}$ The Supplemental Materials include overall and moderator results when including both positive and mixed interventions.

After this exclusion of effect sizes for negative and mixed interventions, interventions had a significant positive effect on climate change attitudes, $n=69, k=312, g=0.08,95 \% \mathrm{CI}$ $[0.05,0.10], 95 \%$ prediction interval $[-0.04,0.19], t(45.93)=7.39, p<.001, I^{2}=31.32, T=0.05$. Appendix A describes the different characteristics of every report included in the meta-analysis, with the full dataset available online (https://osf.io/q7jhe/).

\subsection{Moderators}

\subsubsection{Political Affiliation}

To test political affiliation as a moderator of intervention effectiveness, we separated effect sizes by political group (namely liberals, conservatives, and moderates) resulting in 905 effect sizes across 67 studies (see Table 2). ${ }^{10}$ Political affiliation was not a significant moderator of intervention effectiveness, $F(30.47)=0.25, p=.78$. In other words, effect sizes did not significantly differ between liberals, conservatives, and moderates.

\subsubsection{Type of Attitude}

\footnotetext{
${ }^{9}$ Although one option could be to simply flip the sign of the effect size of negative interventions, doing so would assume that inducing skepticism is the same as garnering support if one were to merely implement an opposite intervention. However, this assumption of symmetry ignores motivated reasoning processes. For example, if a skeptic sees skeptical information, they may be inclined to believe it (and thus become more skeptical). Flipping the sign assumes that the skeptic would respond positively towards positive information. In reality, there is evidence that the skeptic would discount that positive information (Ditto et al., 2019). Therefore, it is important to keep positive interventions separate from negative ones.

${ }^{10}$ Even though we included measures of both political ideology and political party, we will refer to political groups as liberals, conservatives, and moderates (rather than always repeating both ideologies and parties).
} 
The type of climate change attitude was a significant moderator of effect size (Table 2). Specifically, interventions were significantly less effective on policy beliefs than on any other attitude type. As expected, belief in climate change had the largest estimated intervention effect size, although it was only significantly larger than policy support. Type of climate change attitude did not significantly interact with politics, $F(14.70)=0.79, p=.64$. 

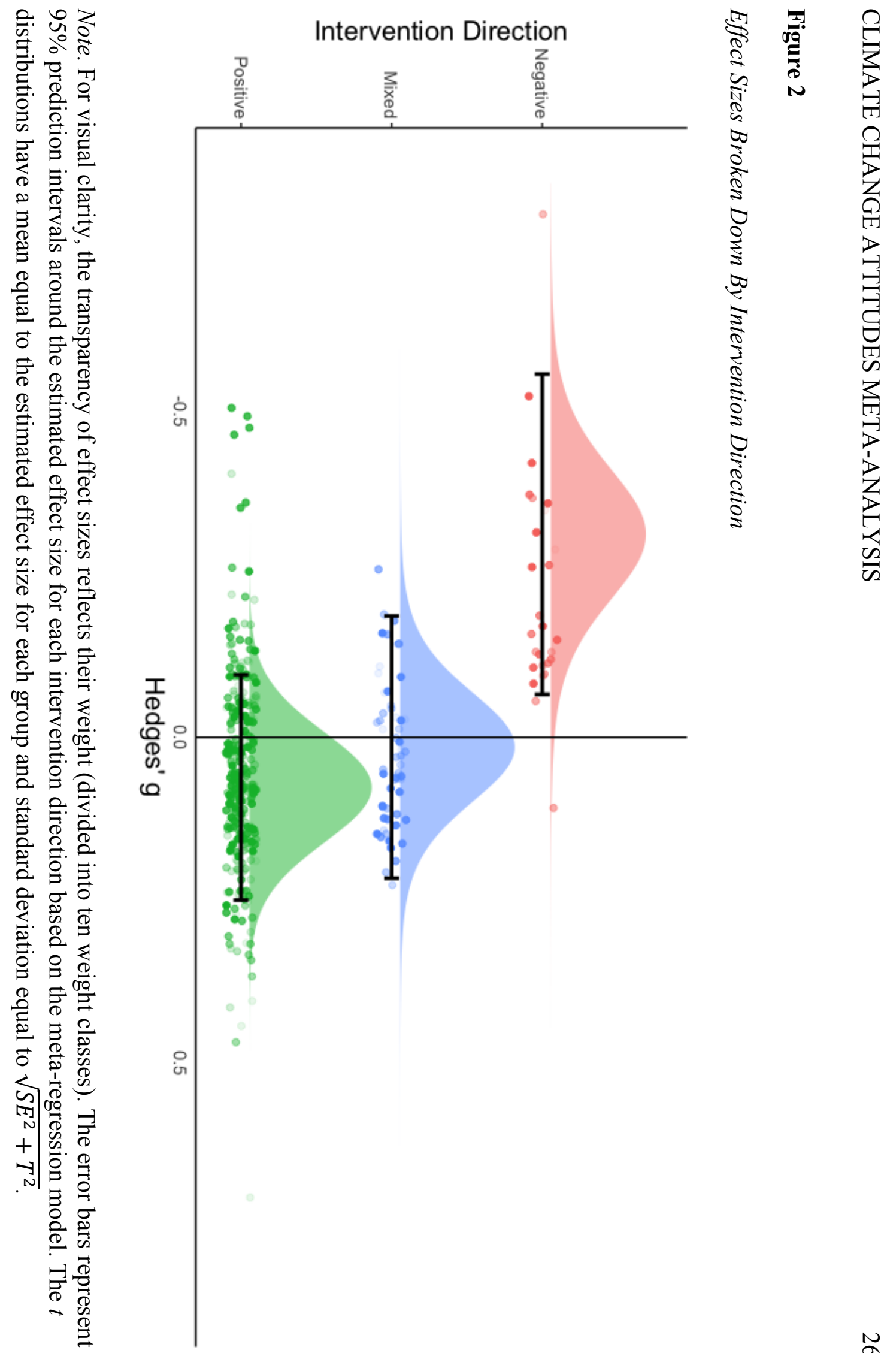


\subsubsection{Type of Intervention}

The effect of interventions on climate change attitudes did not vary significantly as a function of the type of intervention, $F(3.19)=1.48, p=.40$ (Table 2). ${ }^{11}$ Given the large number of coefficients required to test its interaction with politics (i.e., 10 conditions by 3 political groups), we investigated the interaction effect of politics by conducting separate models for each political affiliation. However, because the number of independent studies contributing to each political group by intervention category was small ( 2 for liberals and moderates while 3 for conservatives) and the categories were unbalanced resulting in few degrees of freedom, we report these results in the Supplemental Materials. Although these analyses find no significant evidence of moderation by political affiliation, they have little power to detect it (the Supplemental Materials also include exploratory analyses where we removed intervention types with few studies contributing to them).

Figure 3 depicts effect sizes by type of intervention. Although the inferential conclusions are limited, this graph reveals general trends of effect sizes and how they vary by intervention type. The most promising interventions were those that invoked emotion, decreased psychological distance, and involved religion (see Table 2 for estimated effect sizes for each of these intervention types). In contrast to the other types of intervention, ones focusing on scientific consensus were tested in a larger number of independent samples $(n=20)$. Conducting a separate meta-analysis with just consensus interventions, the effect size was statistically

\footnotetext{
${ }^{11}$ Using the original seven categories, intervention type was a significant moderator, $F(6.32)=4.48, p=.04$. However, none of the individual meta-regression coefficients were significantly different from 0 . All of the coefficients using the original categories were similar to those when using the expanded categories. Similar to the expanded categories, the original seven categories were not a significant moderator for liberals, conservatives, or moderates (Supplemental Materials).
} 
significant and positive, $g=0.09,95 \%$ CI [0.05, 0.13], $p=.004$, and not significantly moderated by political group, $F(6.65)=0.89, p=.45$.

\subsubsection{Type of Sample}

Sample type was not a significant moderator of intervention effects on climate change attitudes, nor did it interact with political affiliation, $F(5.77)=0.51, p=.82$ when samples were consolidated into the five categories described earlier. When using the original coding of sample type (e.g., including YouGov and GfK), sample type was also not a significant moderator of effect sizes, $F(4.81)=1.21, p=.42$. Moreover, representative samples of the U.S. population did not significantly differ from non-representative samples, $b=-0.01,95 \% \mathrm{CI}[-0.05,0.03]$, $t(13.54)=-0.59, p=.56$ 


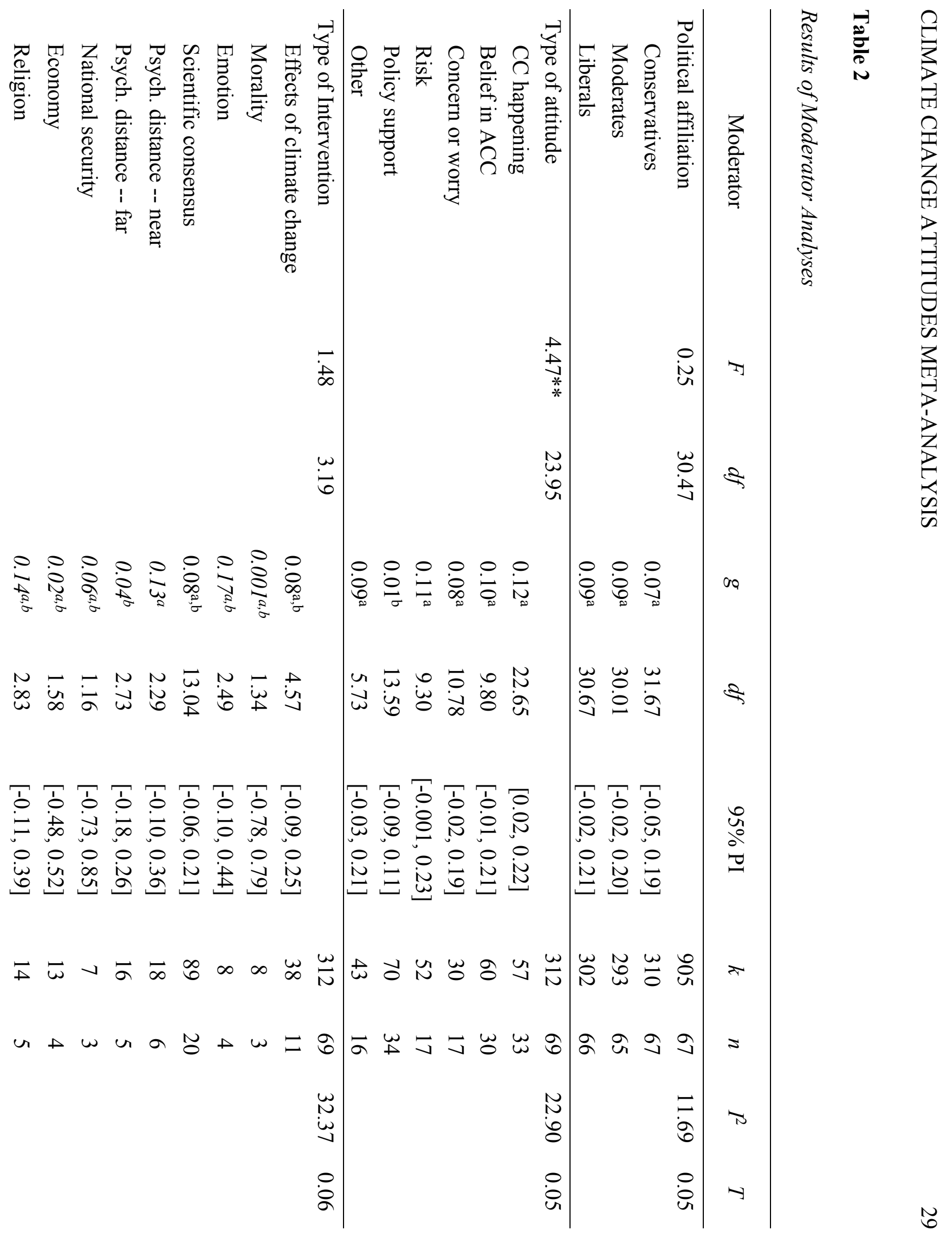




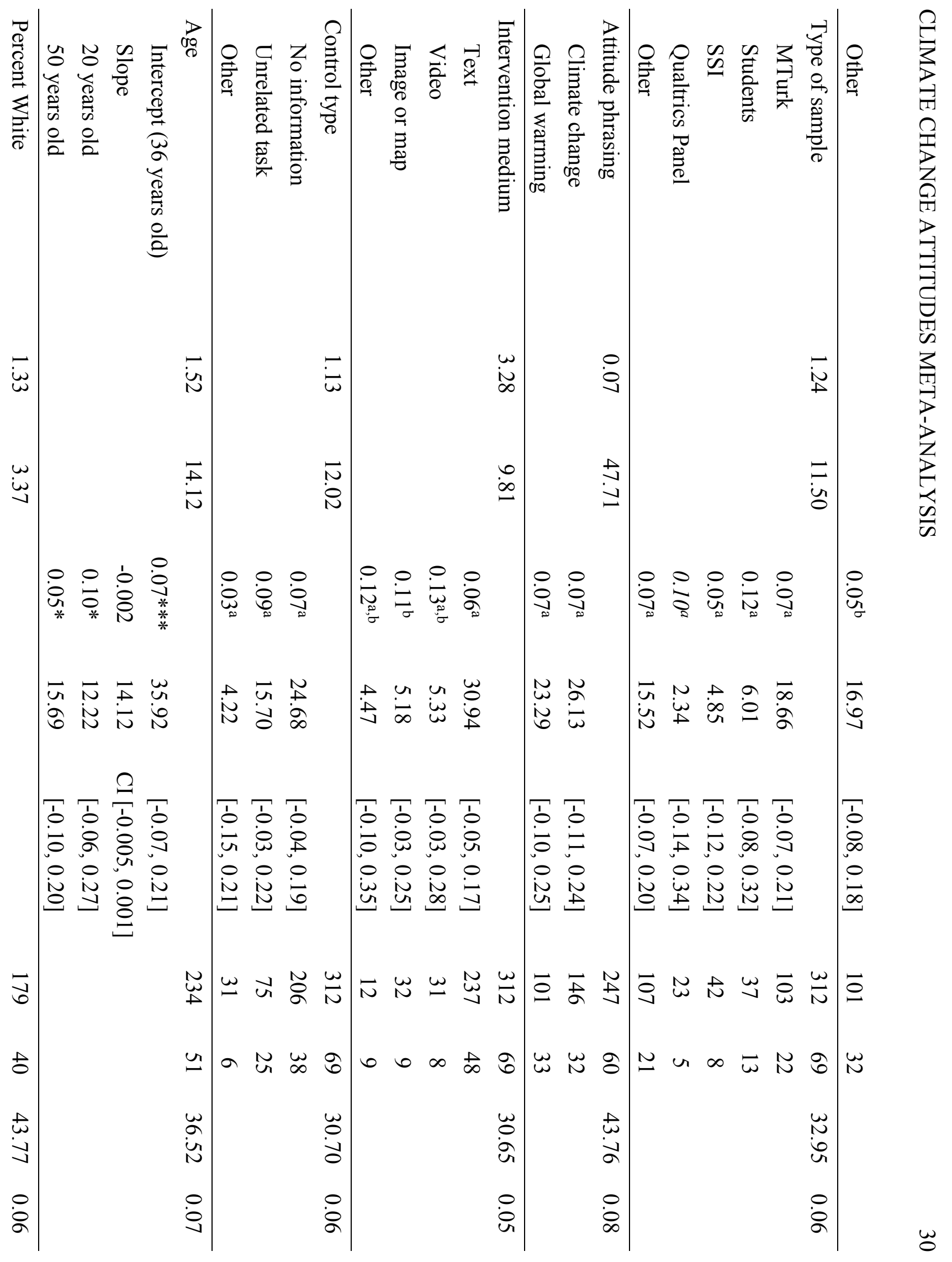




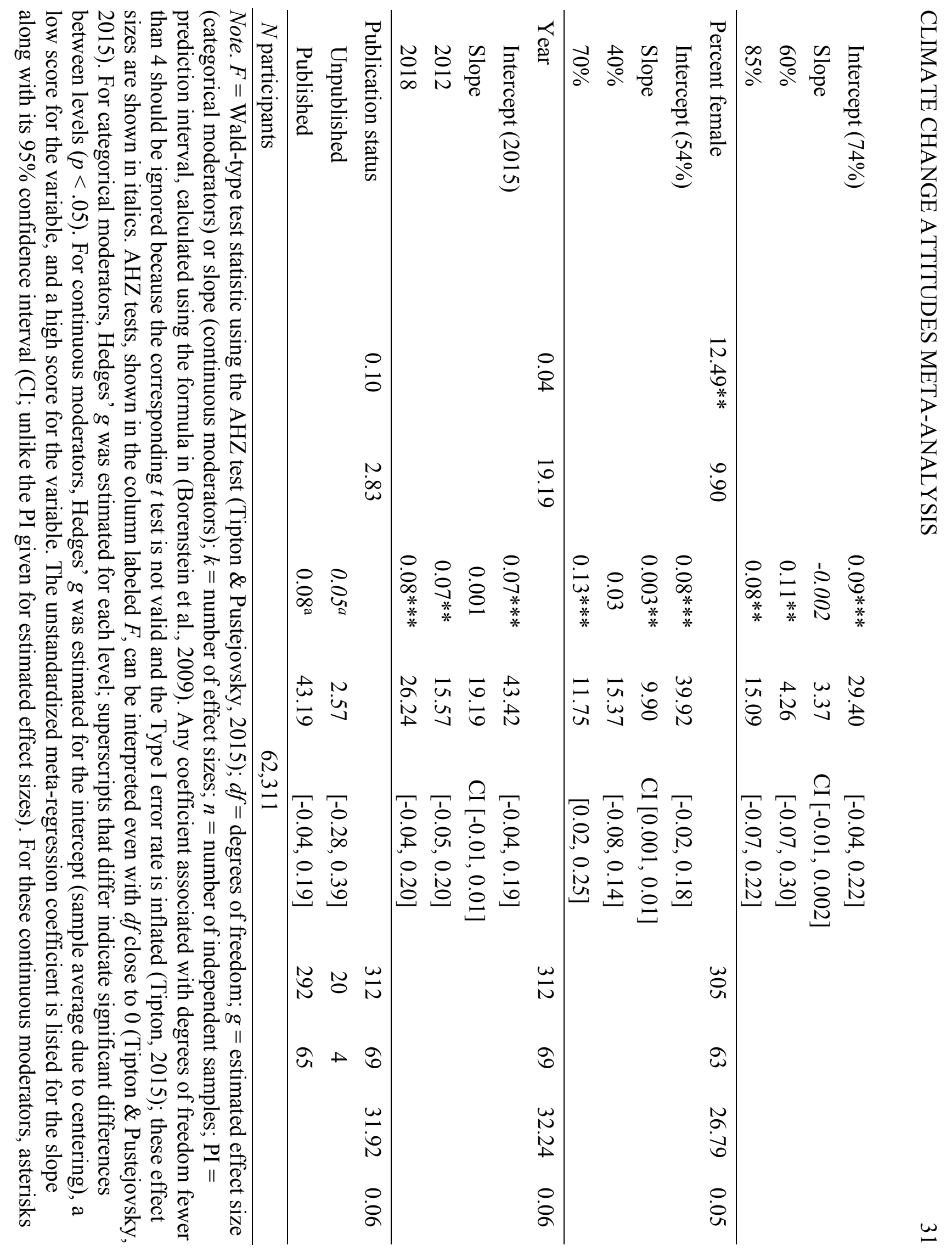




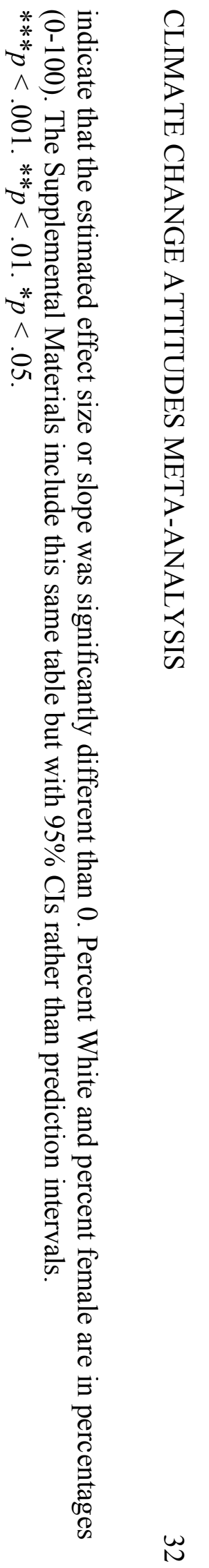



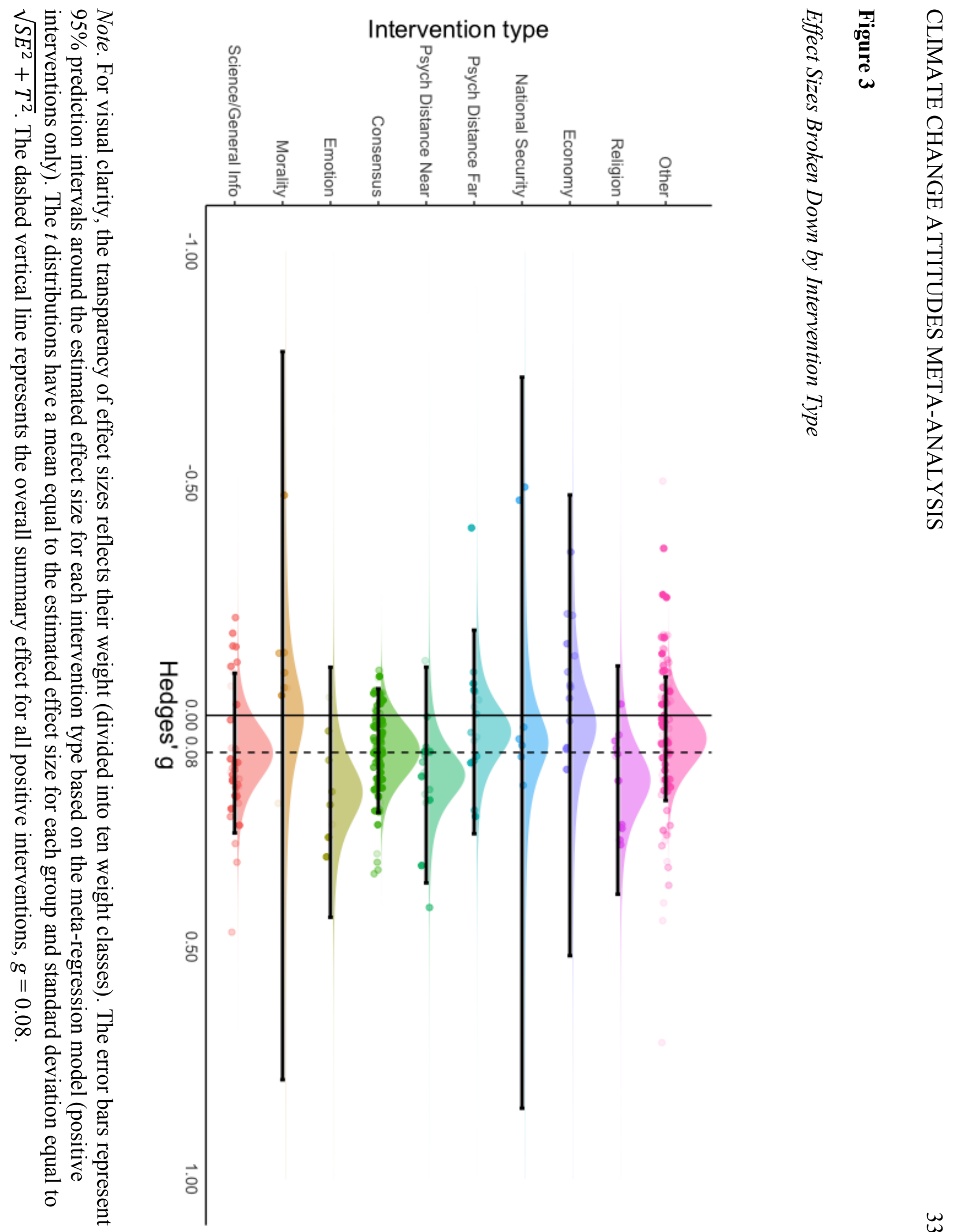


\subsubsection{Other Moderators}

Lastly, we investigated some exploratory and report-level moderators, namely attitude phrasing, intervention medium, control type, age, percent White, percent female, year of data collection, and publication status (Table 2). Effect sizes did not significantly vary by attitude phrasing, namely as climate change or global warming (Table 2). However, political affiliation significantly interacted with phrasing, $F(29.44)=3.32, p=.0499$, such that interventions were marginally more effective for conservatives when global warming was the phrase rather than climate change, $b=0.09,95 \%$ CI [-0.003, 0.19], $p=.06$. For liberals $(b=-0.04,95 \%$ CI $[-0.10$, $0.02], p=.23)$ and moderates $(b=-0.03,95 \% \mathrm{CI}[-0.11,0.06], p=.53)$, interventions were (nonsignificantly) less effective with the phrase global warming compared to climate change. However, none of the comparisons (climate change vs. global warming for liberals, conservatives, or moderates) were statistically significant and the overall interaction is not robust under sensitivity analyses, becoming marginally significant when the assumed within-study correlation was set to 1 (rather than .8).

The only other significant moderator was percentage female (Table 2), where effect sizes were significantly larger in studies with a higher percentage of females in the sample. Based on the meta-regression coefficient, a $10 \%$ increase in females was associated with an increase in Hedges' $g$ of 0.03 .

\subsection{Publication Bias}

To examine the possibility of publication bias in the observed effect sizes, we visually inspected and inferentially tested for asymmetry around funnel plots. We constructed funnel plots using two datasets: one dataset that aggregated dependent effect sizes using the MAd package in R (Del Re \& Hoyt, 2014) to calculate one effect size per independent sample, 
assuming a within-study correlation of .50, and another dataset that ignored dependencies and used all effects (i.e., multiple effect sizes per sample), treating them as if they were independent (excluding mixed and negative interventions as in the main analyses). Figure 4 displays the funnel plot for the aggregated dataset (only one effect size per independent sample), and Figure 5 shows the plot for the full dataset (all effect sizes, ignoring dependencies) with both plots visually seeming symmetrical. We also conducted an Egger regression test using RVE, with standard errors as a predictor of the observed effect sizes (Egger et al., 1997; Rodgers \& Pustejovsky, 2020). The standard error was not a significant predictor of effect sizes using RVE, $b=0.04,95 \%$ CI $[-0.54,0.62], t(23.71)=0.14, p=.89 .{ }^{12}$ Considering both of these methods together, there is little evidence of publication bias in the meta-analyzed studies (see the Supplemental Materials for potential explanations).

\footnotetext{
${ }^{12}$ Pustejovsky and Rodgers (2019) suggest using a modified covariate in place of standard error (assuming balanced sample sizes between control and treatment). An Egger regression test with this modified covariate was also not significant, $b=0.02,95 \% \mathrm{CI}[-0.56,0.61], t(25.06)=0.08, p=.94$.
} 


\section{Figure 4}

Funnel Plot of Aggregated Effect Sizes

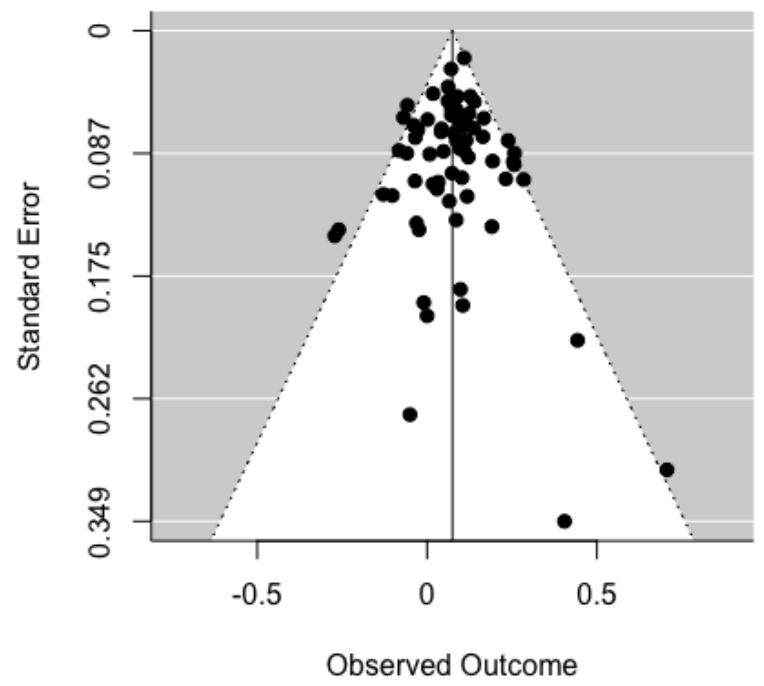

Figure 5

Funnel Plot of All Effect Sizes, Ignoring Dependencies

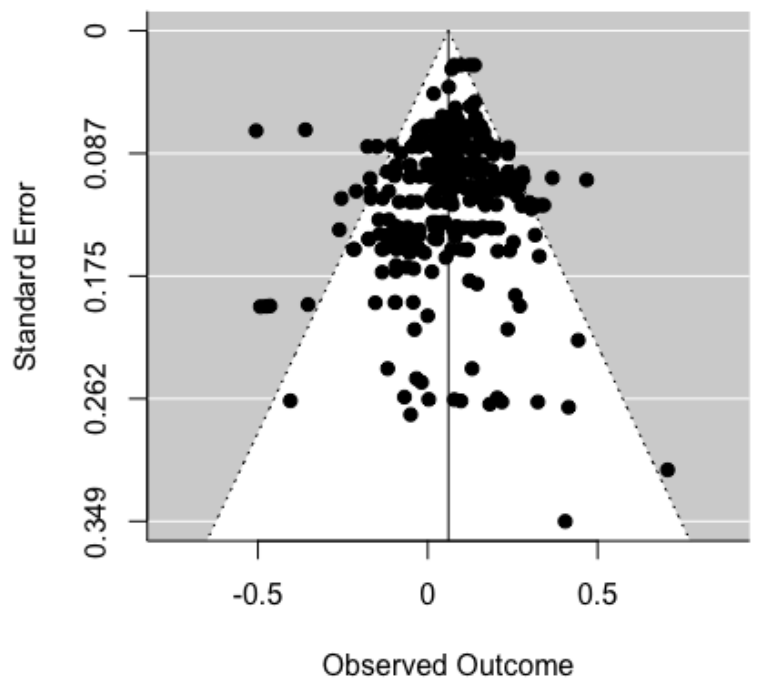




\section{Discussion}

The current meta-analysis quantitatively integrated and reconciled experimental studies that tested interventions to influence climate change attitudes. This meta-analysis brought together 76 independent experiments with 396 total effect sizes that tested an intervention against a control condition to enable a consistent comparison across studies. Addressing climate change by implementing mitigation and adaptation strategies requires a public willingness to take action. Therefore, it is necessary to discover and develop interventions designed to increase the public's belief in, concern for, and endorsement of action on climate change. This metaanalysis provides rigorous insight into those interventions.

\subsection{Interpretations}

Given the nature and goals of studies included in the meta-analysis, some interventions were actually intended to reduce belief in climate change. This direction of intervention -positive, negative, or mixed -- changed both the magnitude and direction of its effect on climate change attitudes. Not surprisingly, interventions intended to reduce beliefs in climate change significantly did so while interventions intended to increase them significantly did so. The negative and positive interventions used similar strategies to influence attitudes (e.g., a news article).

Even though the intervention strategies were similar, effect sizes were much stronger ( $g=$ $-0.29)$ for negative interventions than for positive ones $(g=0.08)$. This finding highlights the relative ease with which people are willing to doubt climate change as opposed to accept it. Given that climate change is an uncertain event with potentially devastating negative consequences, people might tend to cling to information that gives them hope even if that hope is grounded in downplaying the consequences. 
The type of intervention was not a significant moderator of its effect on climate change attitudes and thus we cannot make a statistical claim as to the best intervention. However, by separately estimating summary effects for interventions, we found that emotion, psychological distance (near), and religious interventions displayed the most promise. The small number of effect sizes and limited number of independent samples likely contributed to the nonsignificant moderation by intervention type.

Attitude type was a significant moderator of intervention effectiveness. In particular, attitudes about climate change beliefs appear more malleable than attitudes about climate change policy with a significantly weaker effect on them. This finding was in line with previous research (Hornsey et al., 2016), given that support for policy may imply a cost and would be expected to occur after one accepts climate change. However, beyond implying additional costs, policy beliefs may have a smaller average effect for other reasons. The finding could also be due to the goal of interventions: some interventions may have been targeting belief in climate change, with endorsement of climate policy measured in the study but not as the focal outcome. As a result, these interventions may be better suited for influencing belief but not policy support. Additionally, policy support may be more downstream than belief in climate change. For example, the GBM posits that people's knowledge about scientific agreement influences their belief in and worry about climate change, which in turn increase their support for public action (van der Linden et al., 2015). Policy support may require people to accept the initial facts of climate change and connect how policies solve the problem. There are numerous reasons for the observed tenacity of policy beliefs including that policies imply costs, interventions may not have targeted policies specifically, and policies require more complex knowledge. Whatever the mechanism, these results, especially given variation across studies in other factors, suggest that it 
is more difficult to influence people's attitudes about supporting a climate change policy than to persuade them that climate change exists.

Political affiliation was not a significant moderator of intervention effectiveness, indicating that interventions were similarly effective for liberals, conservatives, and moderates. Although research on motivated reasoning might predict that conservatives would be particularly unaffected by the typical positive intervention (e.g., not one targeted at conservatives), such interventions were relatively ineffective across the political spectrum. Conservatives may have been dismissive of positive interventions on average (leading to low effectiveness) and liberals may have exhibited a ceiling effect (with strong climate change attitudes even before interventions). Or, directionally motivated reasoning processes may have played little role and positive interventions were simply ineffective for all political groups (or other unknown factors were involved). Importantly, many of the interventions used tailored strategies to reduce motivated reactions to messages, but the current meta-analysis had limited statistical power to detect how the effect of various intervention types were differentially impacted by political affiliation. The match between individual motivation (e.g., accuracy) and message type is important (Bayes et al., 2020) yet difficult to pinpoint given the variety of studies in this metaanalysis. Nevertheless, some interventions may be useful not because they lead to large changes in attitudes, but because they have consistent positive effects for all political groups without causing backfire (e.g., consensus messages).

Although we were able to test political affiliation as a moderator of intervention effectiveness, inferences from its nonsignificant findings are limited. In particular, the paucity of studies in most moderator categories and the complexity of their modeling in the meta-analysis means that some types of interventions may have been particularly effective or ineffective for 
certain partisans but there were not enough independent studies to detect significant differences.

As a result, an overall effect of political affiliation may be hidden by its entangled relation with intervention type. ${ }^{13}$

\subsection{Implications}

\subsubsection{Small Impact of Intervention}

The results have a number of relevant implications for researchers and policymakers interested in climate change communication and related fields. First and foremost, the results point to the limited effectiveness to date of interventions to promote positive climate change attitudes in comparison to a control condition. The summary effect was small with few significant moderators of it. The summary effect was smaller than that of a more specific metaanalysis on framing and climate change attitudes $(S M D=0.17$; Li \& Su, 2018), smaller than that of a meta-analysis on framing and political attitudes (positive frames vs. control: $d=0.24$; Amsalem \& Zoizner, 2020), and it was much smaller than that of a meta-analysis on interventions targeting pro-environmental behaviors ( $g=0.45$; Osbaldiston \& Schott, 2012). Visualized in Figure 6, an effect of $g=0.08$ indicates that most of the control and intervention groups overlap and the two distributions look nearly identical. Moreover, the intervention effects were consistently small (as illustrated by the summary effect's $95 \%$ prediction interval [-0.04, 0.19]). Based on these results, it appears that many of the interventions included in this metaanalysis -- specifically one-time messages in experimental settings -- are not an effective approach to changing climate change attitudes.

\footnotetext{
${ }^{13}$ When interpreting the results of political affiliation, it is important to note that sample sizes for conservative effect sizes were much smaller than those for liberal effect sizes (see full data at: https://osf.io/q7jhe/), leading to increased variance for conservative effect sizes. This added noise may have made it more difficult to detect significant differences between political groups and significant interactions with political affiliation. The null results here do not point to a lack of a political effect, but rather are inconclusive.
} 
These relatively small effects, however, do not imply that interventions for climate change attitudes are never effective or are never meaningful. Interventions on climate change attitudes may be effective in specific situations, but there are not enough of those studies for their effects to stand out statistically (e.g., morality interventions are promising but only three independent studies were available for analysis). Moreover, some promising interventions do not include control conditions and thus did not quality for this meta-analysis (e.g., Campbell \& Kay, 2014).

Additionally, the current meta-analysis was restricted to one-time messages. It is not surprising that a single message in an experimental setting might have little sway on entrenched political attitudes. In contrast, effects could accumulate over time or after repeated exposure and eventually have a meaningful impact (Funder \& Ozer, 2019). However, this possibility of additive effects is only speculative as repeated exposure could also lead to decreased effectiveness over time or a return to baseline after an initial attitude change. Another possibility is that interventions with relatively small effects could be practically important if they are seen by hundreds of thousands or millions of people, which could reasonably occur for some of the interventions in this meta-analysis (e.g., a video demonstrating the $97 \%$ scientific consensus -and used as an intervention in Brewer and McKnight, 2017 -- has over 8.6 million views as of late January, 2021; LastWeekTonight, 2014). For example, a Cohen's $d$ of 0.08 means that about 43 people need to receive a treatment for the treatment group to have more favorable outcomes than the control group (Magnusson, 2020). ${ }^{14}$ Given the widespread availability of online

\footnotetext{
${ }^{14}$ Number needed to treat (NNT) relies on the assumption that $20 \%$ of the control group have favorable outcomes (e.g., believe that climate change is happening). NNT changes drastically with different assumptions about the control group (e.g., from 427 assuming 1\% or 31 assuming 50\%). Given current public opinion data (Leiserowitz, Maibach, Rosenthal, Kotcher, Bergquist, et al., 2020), we might expect about $73 \%$ of the control group to believe in climate change (resulting in 39 NNT) with that percentage decreasing with attitudes like policy support. However, the use of NNT has been criticized (e.g., Stang et al., 2010) and relying on it assumes dichotomous climate change attitudes (e.g., belief/non-belief or support/opposition) when these attitudes are generally treated continuously.
} 
information, treating an additional 43 people would be quite easy and could amount to an impactful intervention with a large number of people (e.g., a social media post with millions of views). However, by largely relying on aggregate data, this meta-analysis is unable to test whether interventions across a large number of people would translate to uniformly small changes for everyone or instead meaningful changes for a few people. These different effects could impact how meaningful interventions are when applied to a large number of people. While the small summary effect does not imply that interventions are never effective, this meta-analysis lacks data on the effectiveness of repeated or large-scale interventions and thus these discussions around potentially meaningful effects are speculative.

\section{Figure 6}

Simulated Control and Treatment Distributions for an Effect of 0.08

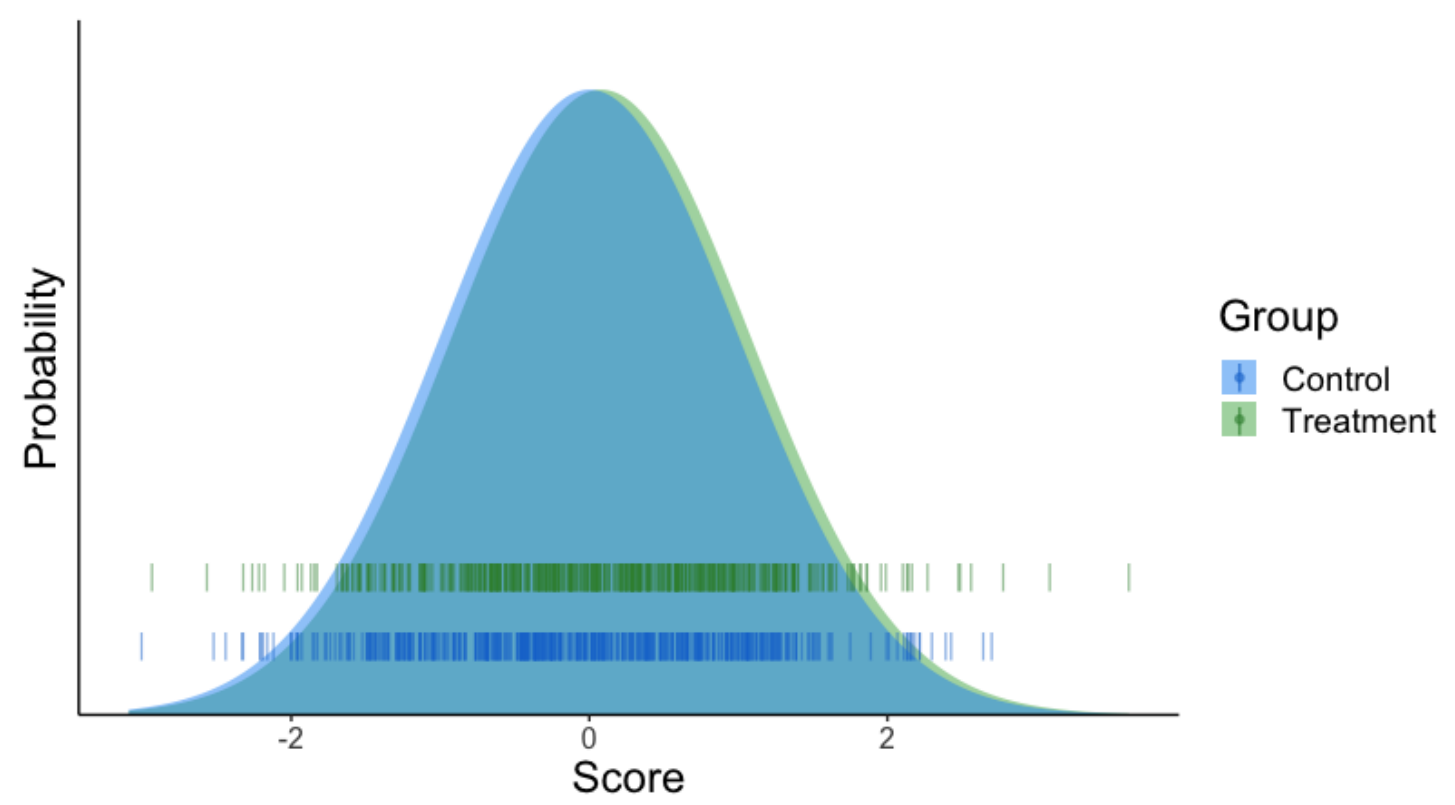

Note. The control and intervention groups each include 500 observations, and each have a standard deviation of 1 . The control group has a mean of 0 and the intervention group has a mean of 0.08 . 


\subsubsection{Asymmetry of Skepticism and Support}

Given the finding of asymmetry of negative and positive interventions, policymakers and those communicating the science of climate change should be cautious when discussing climate science. One potential solution is to provide information about the scientific consensus. Although doing so as an intervention itself has limited effectiveness (our current results show an effect size of $g=0.09$ for consensus manipulations), consensus messaging is a valuable tool to inoculate the public against misinformation (Cook et al., 2017; Maertens et al., 2020). Moreover, our asymmetry finding implies that positive interventions have a higher standard than negative ones: a generic message about the limited effects of climate change may be enough to induce skepticism, but a generic message about the extensive effects of climate change will probably not be a strong positive influence on attitudes. As a result, interventions need to be especially persuasive and tailored to an audience's values (Druckman \& McGrath, 2019) to increase supportive attitudes toward climate change and ultimately influence policy support.

\subsubsection{Policy Support is Difficult to Influence}

Our results indicate that climate change belief is much easier to influence than support for climate change policy. Unfortunately, policy support is more important than belief, with belief generally seen as only instrumentally important to drive support for climate policies. Even if interventions were not effective for beliefs, meaningfully moving the dial in policy support would produce important implications for policymakers. For example, P. S. Hart and Feldman (2018) found that people were more receptive to policy when it was framed around air pollution rather than climate change, suggesting that there may be ways to garner policy support among climate skeptics without changing their minds about the existence of climate change. Although it is difficult to sway policy attitudes, there may be ways to influence policy support without first 
changing belief in climate change. Targeted interventions for specific policies may be particularly effective for meaningful climate action (e.g., highlighting policy effectiveness; Reynolds et al., 2020).

\subsection{Limitations}

These important implications and directions from our meta-analysis should be seen in light of three main limitations.

First, this meta-analysis combined a wide range of experimental studies, each with its own goals and focus. Synthesizing this broad range of studies permits evaluating the field as a whole and examining the myriad types of interventions that would have been excluded from a narrower meta-analysis. Reflecting this variation, several studies included in the meta-analysis focused on mediation or moderation of intervention effectiveness rather than its main effect. The authors of those studies might not have hypothesized a main effect of interventions on climate change attitudes. Only looking at the main effects of interventions limits the ability of the current meta-analysis to examine how fine-grained intervention characteristics (e.g., combinations of interventions, different response scales) explain variation in effect sizes. Differences between study design and our meta-analytic design highlight that "intervention effectiveness" differs depending on context. Although the summary effect size was small when compared to effects found in other meta-analyses, there are a variety of factors that contribute to intervention effectiveness, many of which were unable to be analyzed in the current meta-analysis.

Second, the generalizability of findings should be considered when applying them. For example, it was not possible to include the entire population of qualifying studies in our metaanalysis. Although we attempted to obtain every study that fit its inclusion criteria, we were unable to retrieve data from 12 of the qualifying reports and inevitably missed some reports, as is 
a common concern in meta-analysis more generally. Although unpublished studies are always more likely omitted than published ones, our three tests of publication bias indicate that the results are robust to omitted studies. Also related to generalizability, the current meta-analysis was restricted to studies conducted with samples from the United States due to the focus on climate change within the American political landscape. Interventions might have larger effects in other countries where climate change attitudes are less politicized (Hornsey et al., 2018). If climate change is an apolitical issue in another country, then perhaps attitudes are more easily swayed and thus interventions would be more effective in that country. The current results suggest that, at least within the politicized context of the United States, climate change attitudes are difficult to change.

Additionally, the meta-analysis excluded studies focused on behavior thereby limiting its generalizability to attitudes only. Although behavioral outcomes would have been interesting and important to investigate, including them would have substantially expanded the scope of the meta-analysis. For example, many environmental behaviors are indirectly related to climate change (e.g., recycling, household energy use). Creating a distinction between climate change and the environment in general was easier for attitudes (with many involving face-valid questions about one's perceptions of climate change) than for behaviors, where the interconnected nature of climate systems means that many behaviors can be eventually linked to climate. Lastly, the current meta-analysis only included one-time messages. As discussed earlier, repeated interventions could exhibit a cumulative effect and increase the practical impact of a very small effect size. However, the current meta-analysis is unable to empirically test this possibility. 
Third, we focused on attitudes specific to climate change, excluding attitudes toward the environment in general or related environmental topics. However, attitudes specific to climate change still include a very broad range of measures and constructs. For example, outcomes ranged from predicted levels of sea-ice to the personal importance of climate change to foreign countries' responsibility for addressing climate change. Although conceptually reasonable to combine as climate change attitudes, doing so has the potential to convolute what the calculated effect sizes estimate. While including attitude type as a moderator helped demonstrate how effect size estimates varied due to them, the overall summary effect estimates much more broadly and should be interpreted with caution given the significant moderator by type of attitude.

\subsection{Future Directions}

Future research should examine how and why attitudes about climate change are more sensitive to negative than positive messages about it. Moreover, future research could examine ways of offsetting this increased malleability to skeptical messages about climate change. Doing so would reduce the potential for increased uncertainty around climate science that so often sparks skepticism (e.g., Dunlap \& Jacques, 2013).

In addition, our findings indicate that interventions were more effective if they were conducted in samples with a higher percentage of participants identifying as female and that attitude phrasing interacted with political affiliation. While we did not develop predictions about gender or attitude phrasing, the results may spark interest in future research on the topics. For example, a substantial body of research has investigated gender differences in environmental concern (e.g., Bloodhart \& Swim, 2020; McCright, 2010). Future research could continue to investigate not only gender differences in climate change beliefs but also in differential response to interventions. In addition, we found that interventions were slightly more effective for 
conservatives when they used the term "global warming" than "climate change" (with the opposite pattern for liberals and moderates). These results add to a growing body of work on this topic (e.g., Soutter \& Mõttus, 2020) and pose a new way of studying responses to the terms, namely comparing if interventions are differentially effective between them.

Our findings also suggest that research should investigate ways of making climate policy palatable. For example, previous research shows that avoiding the term "tax" is useful for garnering policy support (Hardisty et al., 2010, 2019). Additionally, framing policy as being supported by the ingroup may increase support, although findings using this strategy are somewhat mixed (Bolsen et al., 2019b; Fielding et al., 2020; Zhou, 2016). Future research should continue to focus on policy support as an intervention outcome (for a review, see Kyselá et al., 2019) and consider testing different types of policy support (e.g., word framing) along with the effectiveness of different types of interventions (e.g., ingroup support) on these more nuanced aspects of policy support.

Finally, the current meta-analysis is one of the first to organize the varied interventions on climate change attitudes into specific categories. While other unnamed categories of interventions may remain, future research could build upon past work in the same category identified in this project to help facilitate future attempts to integrate and reconcile this growing - and potentially fracturing -- area of research.

\subsection{Conclusion}

This meta-analysis reveals important insights about climate change attitudes and interventions to promote them by combining nearly a decade of diverse studies. First, it is easier to shift people towards skepticism of climate change than belief in it. Second, interventions to promote these positive attitudes show little sign of effectiveness when focusing on their main 
effects. Third, the type of climate change attitude matters more than the type of intervention.

Fourth, climate change beliefs are less resistant to intervention than support for climate change policies. Taken together, we know more now about this topical and timely challenge that should inform both future research and policy. 


\section{References $^{15}$}

*Albertson, B., \& Busby, J. W. (2015). Hearts or minds? Identifying persuasive messages on climate change. Research \& Politics, 2(1), 1-9. https://doi.org/10.1177/2053168015577712

Amsalem, E., \& Zoizner, A. (2020). Real, but limited: A meta-analytic assessment of framing effects in the political domain. British Journal of Political Science, 1-17. https://doi.org/10.1017/S0007123420000253

*Anderson, A. A., \& Becker, A. B. (2018). Not just funny after all: Sarcasm as a catalyst for public engagement with climate change. Science Communication, 40(4), 524-540. https://doi.org/10.1177/1075547018786560

*Bakaki, Z., \& Bernauer, T. (2017). Do global climate summits influence public awareness and policy preferences concerning climate change? Environmental Politics, 26(1), 1-26. https://doi.org/10.1080/09644016.2016.1244964

*Baumer, E. P. S., Polletta, F., Pierski, N., \& Gay, G. K. (2017). A simple intervention to reduce framing effects in perceptions of global climate change. Environmental Communication, 11(3), 289-310. https://doi.org/10.1080/17524032.2015.1084015

Bayes, R., Druckman, J. N., Goods, A., \& Molden, D. C. (2020). When and how different motives can drive motivated political reasoning. Political Psychology, 41(5), 1031-1052. https://doi.org/10.1111/pops. 12663

Benjamin, D., Por, H.-H., \& Budescu, D. (2017). Climate change versus global warming: Who is susceptible to the framing of climate change? Environment and Behavior, 49(7), 745-770. https://doi.org/10.1177/0013916516664382

Berinsky, A. J., Huber, G. A., \& Lenz, G. S. (2012). Evaluating online labor markets for

\footnotetext{
${ }^{15}$ References marked with an asterisk indicate studies included in the meta-analysis.
} 
experimental research: Amazon.com's Mechanical Turk. Political Analysis, 20(3), 351368. https://doi.org/10.1093/pan/mpr057

Bloodhart, B., \& Swim, J. K. (2020). Sustainability and consumption: What's gender got to do with it? Journal of Social Issues, 76(1), 101-113. https://doi.org/10.1111/josi.12370

Boas, T. C., Christenson, D. P., \& Glick, D. M. (2020). Recruiting large online samples in the United States and India: Facebook, Mechanical Turk, and Qualtrics. Political Science Research and Methods, 8(2), 232-250. https://doi.org/10.1017/psrm.2018.28

*Bolsen, T., \& Druckman, J. N. (2018). Do partisanship and politicization undermine the impact of a scientific consensus message about climate change? Group Processes \& Intergroup Relations, 21(3), 389-402. https://doi.org/10.1177/1368430217737855

*Bolsen, T., Kingsland, J., \& Palm, R. (2018). The impact of frames highlighting coastal flooding in the USA on climate change beliefs. Climatic Change, 147, 359-368. https://doi.org/10.1007/s10584-018-2143-0

*Bolsen, T., Leeper, T. J., \& Shapiro, M. A. (2014). Doing what others do: Norms, science, and collective action on global warming. American Politics Research, 42(1), 65-89. https://doi.org/10.1177/1532673X13484173

*Bolsen, T., Palm, R., \& Kingsland, J. T. (2019a). Counteracting climate science politicization with effective frames and imagery. Science Communication, 41(2), 147-171. https://doi.org/10.1177/1075547019834565

Bolsen, T., Palm, R., \& Kingsland, J. T. (2019b). The impact of message source on the effectiveness of communications about climate change. Science Communication, 41(4), 464-487. https://doi.org/10.1177/1075547019863154

Bord, R. J., O'Connor, R. E., \& Fisher, A. (2000). In what sense does the public need to 
understand global climate change? Public Understanding of Science, 9(3), 205-218. https://doi.org/10.1088/0963-6625/9/3/301

Borenstein, M., Hedges, L. V., Higgins, J. P. T., \& Rothstein, H. R. (2009). Introduction to metaanalysis. Wiley.

*Brewer, P. R., \& McKnight, J. (2015). Climate as comedy: The effects of satirical television news on climate change perceptions. Science Communication, 37(5), 635-657. https://doi.org/10.1177/1075547015597911

*Brewer, P. R., \& McKnight, J. (2017). “A statistically representative climate change debate”: Satirical television news, scientific consensus, and public perceptions of global warming. Atlantic Journal of Communication, 25(3), 166-180.

https://doi.org/10.1080/15456870.2017.1324453

Brügger, A. (2020). Understanding the psychological distance of climate change: The limitations of construal level theory and suggestions for alternative theoretical perspectives. Global Environmental Change, 60, 102023. https://doi.org/10.1016/j.gloenvcha.2019.102023

*Budescu, D. V., Por, H.-H., \& Broomell, S. B. (2012). Effective communication of uncertainty in the IPCC reports. Climatic Change, 113, 181-200. https://doi.org/10.1007/s10584-0110330-3

Buhrmester, M., Kwang, T., \& Gosling, S. D. (2011). Amazon's Mechanical Turk: A new source of inexpensive, yet high-quality, data? Perspectives on Psychological Science, 6(1), 3-5. https://doi.org/10.1177/1745691610393980

Campbell, T. H., \& Kay, A. C. (2014). Solution aversion: On the relation between ideology and motivated disbelief. Journal of Personality and Social Psychology, 107(5), 809-824. https://doi.org/10.1037/a0037963 
*Carrico, A. R., Truelove, H. B., Vandenbergh, M. P., \& Dana, D. (2015). Does learning about climate change adaptation change support for mitigation? Journal of Environmental Psychology, 41, 19-29. https://doi.org/10.1016/j.jenvp.2014.10.009

Casler, K., Bickel, L., \& Hackett, E. (2013). Separate but equal? A comparison of participants and data gathered via Amazon's MTurk, social media, and face-to-face behavioral testing. Computers in Human Behavior, 29(6), 2156-2160.

https://doi.org/10.1016/j.chb.2013.05.009

Chapman, D. A., Lickel, B., \& Markowitz, E. M. (2017). Reassessing emotion in climate change communication. Nature Climate Change, 7, 850-852. https://doi.org/10.1038/s41558-017$0021-9$

*Chu, H., \& Yang, J. Z. (2018). Taking climate change here and now - mitigating ideological polarization with psychological distance. Global Environmental Change, 53, 174-181. https://doi.org/10.1016/j.gloenvcha.2018.09.013

*Clarke, E. J. R., Klas, A., Stevenson, J., \& Kothe, E. J. (2019). The role of late-night infotainment comedy in communicating climate change consensus. PsyArXiv. https://doi.org/10.31234/osf.io/ufg9r

*Clayton, S., Koehn, A., \& Grover, E. (2013). Making sense of the senseless: Identity, justice, and the framing of environmental crises. Social Justice Research, 26, 301-319. https://doi.org/10.1007/s11211-013-0185-z

Clifford, S., Jewell, R. M., \& Waggoner, P. D. (2015). Are samples drawn from Mechanical Turk valid for research on political ideology? Research \& Politics, 2(4), 1-9. https://doi.org/10.1177/2053168015622072

*Cook, J., \& Lewandowsky, S. (2016). Rational irrationality: Modeling climate change belief 
polarization using bayesian networks. Topics in Cognitive Science, 8(1), 160-179. https://doi.org/10.1111/tops.12186

*Cook, J., Lewandowsky, S., \& Ecker, U. K. H. (2017). Neutralizing misinformation through inoculation: Exposing misleading argumentation techniques reduces their influence. PLoS ONE, 12(5), e0175799. https://doi.org/10.1371/journal.pone.0175799

Crowson, H. M. (2009). Are all conservatives alike? A study of the psychological correlates of cultural and economic conservatism. The Journal of Psychology, 143(5), 449-463. https://doi.org/10.3200/JRL.143.5.449-463

Crump, M. J. C., McDonnell, J. V., \& Gureckis, T. M. (2013). Evaluating Amazon's Mechanical Turk as a tool for experimental behavioral research. PLoS ONE, 8(3), e57410. https://doi.org/10.1371/journal.pone.0057410

*Cruz, S. M. (2019). Lateral attitude change on environmental issues: Implications for the climate change debate. Climatic Change, 156, 151-169. https://doi.org/10.1007/s10584019-02474-X

Del Re, A. C., \& Hoyt, W. T. (2014). MAd: Meta-analysis with mean differences (R package version 0.8-2). https://cran.r-project.org/package=MAd

*Deryugina, T., \& Shurchkov, O. (2016). The effect of information provision on public consensus about climate change. PLoS ONE, 11(4), e0151469. https://doi.org/10.1371/journal.pone.0151469

Ditto, P. H., Liu, B. S., Clark, C. J., Wojcik, S. P., Chen, E. E., Grady, R. H., Celniker, J. B., \& Zinger, J. F. (2019). At least bias is bipartisan: A meta-analytic comparison of partisan bias in liberals and conservatives. Perspectives on Psychological Science, 14(2), 273-291. https://doi.org/10.1177/1745691617746796 
Ditto, P. H., \& Lopez, D. F. (1992). Motivated skepticism: Use of differential decision criteria for preferred and nonpreferred conclusions. Journal of Personality and Social Psychology, 63(4), 568-584. https://doi.org/10.1037/0022-3514.63.4.568

*Dixon, G., Hmielowski, J., \& Ma, Y. (2017). Improving climate change acceptance among U.S. conservatives through value-based message targeting. Science Communication, 39(4), 520534. https://doi.org/10.1177/1075547017715473

Dixon, G., Hmielowski, J., \& Ma, Y. (2019). More evidence of psychological reactance to consensus messaging: A response to van der Linden, Maibach, and Leiserowitz (2019). Environmental Communication, 1-7. https://doi.org/10.1080/17524032.2019.1671472

Druckman, J. N., \& Kam, C. D. (2011). Students as experimental participants: A defense of the “narrow data base.” In J. N. Druckman, D. P. Green, J. H. Kuklinski, \& A. Lupia (Eds.), Cambridge handbook of experimental political science. Cambridge University Press.

Druckman, J. N., \& McGrath, M. C. (2019). The evidence for motivated reasoning in climate change preference formation. Nature Climate Change, 9, 111-119. https://doi.org/10.1038/s41558-018-0360-1

*Drummond, A., Palmer, M. A., \& Sauer, J. D. (2016). Enhancing endorsement of scientific inquiry increases support for pro-environment policies. Royal Society Open Science, 3(9), 160360. https://doi.org/10.1098/rsos.160360

Dunlap, R. E., \& Jacques, P. J. (2013). Climate change denial books and conservative think tanks. American Behavioral Scientist, 57(6), 699-731. https://doi.org/10.1177/0002764213477096

Egger, M., Smith, G. D., Schneider, M., \& Minder, C. (1997). Bias in meta-analysis detected by a simple, graphical test. $B M J, 315(7109), 629-634$. 
https://doi.org/10.1136/bmj.315.7109.629

Ehret, P. J., Sparks, A. C., \& Sherman, D. K. (2017). Support for environmental protection: An integration of ideological-consistency and information-deficit models. Environmental Politics, 26(2), 253-277. https://doi.org/10.1080/09644016.2016.1256960

Everett, J. A. C. (2013). The 12 item social and economic conservatism scale (SECS). PLoS ONE, 8(12), e82131. https://doi.org/10.1371/journal.pone.0082131

*Feinberg, M., \& Willer, R. (2013). The moral roots of environmental attitudes. Psychological Science, 24(1), 56-62. https://doi.org/10.1177/0956797612449177

Fielding, K. S., Hornsey, M. J., \& Swim, J. K. (2014). Developing a social psychology of climate change. European Journal of Social Psychology, 44(5), 413-420. https://doi.org/10.1002/ejsp.2058

Fielding, K. S., Hornsey, M. J., Thai, H. A., \& Toh, L. L. (2020). Using ingroup messengers and ingroup values to promote climate change policy. Climatic Change, 158, 181-199. https://doi.org/10.1007/s10584-019-02561-z

Fisher, Z., \& Tipton, E. (2015). robumeta: An R-package for robust variance estimation in metaanalysis. http://arxiv.org/abs/1503.02220

Funder, D. C., \& Ozer, D. J. (2019). Evaluating effect size in psychological research: Sense and nonsense. Advances in Methods and Practices in Psychological Science, 2(2), 156-168. https://doi.org/10.1177/2515245919847202

Garrett, R. K. (2009). Echo chambers online?: Politically motivated selective exposure among Internet news users. Journal of Computer-Mediated Communication, 14(2), 265-285. https://doi.org/10.1111/j.1083-6101.2009.01440.x

Gauchat, G. (2012). Politicization of science in the public sphere. American Sociological Review, 
77(2), 167-187. https://doi.org/10.1177/0003122412438225

*Gehlbach, H., Robinson, C. D., \& Vriesema, C. C. (2019). Leveraging cognitive consistency to nudge conservative climate change beliefs. Journal of Environmental Psychology, 61, 134137. https://doi.org/10.1016/j.jenvp.2018.12.004

Gillis, J., \& Popovich, N. (2017, June 6). In Trump country, renewable energy is thriving. The New York Times. https://www.nytimes.com/2017/06/06/climate/renewable-energy-push-isstrongest-in-the-reddest-states.html

*Goldberg, M. H., Gustafson, A., Ballew, M. T., Rosenthal, S. A., \& Leiserowitz, A. (2019). A social identity approach to engaging Christians in the issue of climate change. Science Communication, 41(4), 442-463. https://doi.org/10.1177/1075547019860847

*Goldberg, M. H., van der Linden, S., Ballew, M. T., Rosenthal, S. A., Gustafson, A., \& Leiserowitz, A. (2019). The experience of consensus: Video as an effective medium to communicate scientific agreement on climate change. Science Communication, 41(5), 659673. https://doi.org/10.1177/1075547019874361

*Goldberg, M. H., van der Linden, S., Ballew, M. T., Rosenthal, S. A., \& Leiserowitz, A. (2019). The role of anchoring in judgments about expert consensus. Journal of Applied Social Psychology, 49(3), 192-200. https://doi.org/10.1111/jasp.12576

Graham, J., Haidt, J., \& Nosek, B. A. (2009). Liberals and conservatives rely on different sets of moral foundations. Journal of Personality and Social Psychology, 96(5), 1029-1046. https://doi.org/10.1037/a0015141

Graham, J., Nosek, B. a, Haidt, J., Iyer, R., Koleva, S., \& Ditto, P. H. (2011). Mapping the moral domain. Journal of Personality and Social Psychology, 101(2), 366-385.

https://doi.org/10.1097/00005053-199107000-00016 
*Guisinger, A., \& Saunders, E. N. (2017). Mapping the boundaries of elite cues: How elites shape mass opinion across international issues. International Studies Quarterly, 61(2), 425441. https://doi.org/10.1093/isq/sqx022

Guy, S., Kashima, Y., Walker, I., \& O’Neill, S. (2014). Investigating the effects of knowledge and ideology on climate change beliefs. European Journal of Social Psychology, 44(5), 421-429. https://doi.org/10.1002/ejsp.2039

*Halperin, A., \& Walton, P. (2018). The importance of place in communicating climate change to different facets of the American public. Weather, Climate, and Society, 10, 291-305. https://doi.org/10.1175/WCAS-D-16-0119.1

Hardisty, D. J., Beall, A. T., Lubowski, R., Petsonk, A., \& Romero-Canyas, R. (2019). A carbon price by another name may seem sweeter: Consumers prefer upstream offsets to downstream taxes. Journal of Environmental Psychology, 66, 101342. https://doi.org/10.1016/j.jenvp.2019.101342

Hardisty, D. J., Johnson, E. J., \& Weber, E. U. (2010). A dirty word or a dirty world?: Attribute framing, political affiliation, and query theory. Psychological Science, 21(1), 86-92. https://doi.org/10.1177/0956797609355572

*Hardy, B. W., \& Jamieson, K. H. (2017). Overcoming endpoint bias in climate change communication: The case of Arctic sea ice trends. Environmental Communication, 11(2), 205-217. https://doi.org/10.1080/17524032.2016.1241814

Hart, P. S., \& Feldman, L. (2018). Would it be better to not talk about climate change? The impact of climate change and air pollution frames on support for regulating power plant emissions. Journal of Environmental Psychology, 60, 1-8. https://doi.org/10.1016/j.jenvp.2018.08.013 
Hart, P. S., \& Nisbet, E. C. (2012). Boomerang effects in science communication. Communication Research, 39(6), 701-723. https://doi.org/10.1177/0093650211416646

Hart, W., Albarracín, D., Eagly, A. H., Brechan, I., Lindberg, M. J., \& Merrill, L. (2009). Feeling validated versus being correct: A meta-analysis of selective exposure to information. Psychological Bulletin, 135(4), 555-588. https://doi.org/10.1037/a0015701

Hedges, L. V. (1981). Distribution theory for Glass's estimator of effect size and related estimators. Journal of Educational Statistics, 6(2), 107. https://doi.org/10.2307/1164588

Hedges, L. V., Tipton, E., \& Johnson, M. C. (2010). Robust variance estimation in metaregression with dependent effect size estimates. Research Synthesis Methods, 1(1), 39-65. https://doi.org/10.1002/jrsm.5

Hennes, E. P., Kim, T., \& Remache, L. J. (2020). A goldilocks critique of the hot cognition perspective on climate change skepticism. Current Opinion in Behavioral Sciences, 34, 142-147. https://doi.org/10.1016/j.cobeha.2020.03.009

Hess, D. J., Mai, Q. D., \& Brown, K. P. (2016). Red states, green laws: Ideology and renewable energy legislation in the United States. Energy Research \& Social Science, 11, 19-28. https://doi.org/10.1016/j.erss.2015.08.007

Hornsey, M. J., Harris, E. A., Bain, P. G., \& Fielding, K. S. (2016). Meta-analyses of the determinants and outcomes of belief in climate change. Nature Climate Change, 6, 622626. https://doi.org/10.1038/nclimate2943

Hornsey, M. J., Harris, E. A., \& Fielding, K. S. (2018). Relationships among conspiratorial beliefs, conservatism and climate scepticism across nations. Nature Climate Change, 8 , 614-620. https://doi.org/10.1038/s41558-018-0157-2

*Hu, S., Zheng, X., Zhang, N., \& Zhu, J. (2018). The impact of mortality salience on 
intergenerational altruism and the perceived importance of sustainable development goals. Frontiers in Psychology, 9. https://doi.org/10.3389/fpsyg.2018.01399

IPCC. (2018). Summary for policymakers. In V. Masson-Delmotte, P. Zhai, H.-O. Pörtner, D. Roberts, J. Skea, P. R. Shukla, A. Pirani, W. Moufouma-Okia, C. Péan, R. Pidcock, S. Connors, J. B. R. Matthews, Y. Chen, X. Zhou, M. I. Gomis, E. Lonnoy, T. Maycock, M. Tignor, \& T. Waterfield (Eds.), Global Warming of $1.5^{\circ}$ C. An IPCC Special Report on the impacts of global warming of $1.5^{\circ} \mathrm{C}$ above pre-industrial levels and related global greenhouse gas emission pathways, in the context of strengthening the global response to the threat of climate change. World Meteorological Organization.

*Jamelske, E. (2017). Unpublished data. University of Wisconsin-Eau Claire.

*Jang, S. M. (2013). Framing responsibility in climate change discourse: Ethnocentric attribution bias, perceived causes, and policy attitudes. Journal of Environmental Psychology, 36, 2736. https://doi.org/10.1016/j.jenvp.2013.07.003

*Joireman, J., Barnes Truelove, H., \& Duell, B. (2010). Effect of outdoor temperature, heat primes and anchoring on belief in global warming. Journal of Environmental Psychology, 30(4), 358-367. https://doi.org/10.1016/j.jenvp.2010.03.004

Jost, J. T., Glaser, J., Kruglanski, A. W., \& Sulloway, F. J. (2003). Political conservatism as motivated social cognition. Psychological Bulletin, 129(3), 339-375. https://doi.org/10.1037/0033-2909.129.3.339

Kahan, D. (2017). The "Gateway Belief” illusion: Reanalyzing the results of a scientificconsensus messaging study. Journal of Science Communication, 16(05). https://doi.org/10.22323/2.16050203

Kahan, D. M., Peters, E., Dawson, E. C., \& Slovic, P. (2017). Motivated numeracy and 
enlightened self-government. Behavioural Public Policy, 1(1), 54-86. https://doi.org/10.1017/bpp.2016.2

Karlin, B., Zinger, J. F., \& Ford, R. (2015). The effects of feedback on energy conservation: A meta-analysis. Psychological Bulletin, 141(6), 1205-1227. https://doi.org/10.1037/a0039650

Kees, J., Berry, C., Burton, S., \& Sheehan, K. (2017). An analysis of data quality: Professional panels, student subject pools, and Amazon's Mechanical Turk. Journal of Advertising, 46(1), 141-155. https://doi.org/10.1080/00913367.2016.1269304

Kerr, J. R., \& Wilson, M. S. (2018). Perceptions of scientific consensus do not predict later beliefs about the reality of climate change: A test of the gateway belief model using crosslagged panel analysis. Journal of Environmental Psychology, 59, 107-110. https://doi.org/10.1016/j.jenvp.2018.08.012

*Kotcher, J., Maibach, E., Montoro, M., \& Hassol, S. J. (2018). How Americans respond to information about global warming's health impacts: Evidence from a national survey experiment. GeoHealth, 2(9), 262-275. https://doi.org/10.1029/2018GH000154

Kunda, Z. (1990). The case for motivated reasoning. Psychological Bulletin, 108(3), 480-498. https://doi.org/10.1037/0033-2909.108.3.480

Kyselá, E., Ščasný, M., \& Zvěřinová, I. (2019). Attitudes toward climate change mitigation policies: A review of measures and a construct of policy attitudes. Climate Policy, 19(7), 878-892. https://doi.org/10.1080/14693062.2019.1611534

Lakens, D. (2013). Calculating and reporting effect sizes to facilitate cumulative science: a practical primer for t-tests and ANOVAs. Frontiers in Psychology, 4. https://doi.org/10.3389/fpsyg.2013.00863 
Landis, J. R., \& Koch, G. G. (1977). The measurement of observer agreement for categorical data. Biometrics, 33(1), 159-174. https://doi.org/10.2307/2529310

LastWeekTonight. (2014, May 11). Climate Change Debate: Last Week Tonight with John Oliver $(H B O)$ [Video]. YouTube. https://www.youtube.com/watch?v=cjuGCJJUGsg\&t=2s

Leiserowitz, A., Maibach, E., Rosenthal, S., Kotcher, J., Ballew, M., Bergquist, P., Gustafson, A., Goldberg, M., \& Wang, X. (2020). Politics \& global warming, April 2020. New Haven, CT: Yale Project on Climate Change Communication. https://climatecommunication.yale.edu/publications/politics-global-warming-april-2020/

Leiserowitz, A., Maibach, E., Rosenthal, S., Kotcher, J., Bergquist, P., Ballew, M., Goldberg, M., Gustafson, A., \& Wang, X. (2020). Climate change in the American mind: April, 2020. New Haven, CT: Yale Project on Climate Change Communication. https://climatecommunication.yale.edu/publications/climate-change-in-the-american-mindapril-2020/

Leiserowitz, A., Maibach, E., Roser-Renouf, C., Cutler, M., \& Rosenthal, S. (2017). Trump voters \& global warming. New Haven, CT: Yale Project on Climate Change Communication. https://climatecommunication.yale.edu/publications/trump-voters-globalwarming/

Levay, K. E., Freese, J., \& Druckman, J. N. (2016). The demographic and political composition of Mechanical Turk samples. SAGE Open, 6(1), 1-17. https://doi.org/10.1177/2158244016636433

Lewandowsky, S., \& Oberauer, K. (2016). Motivated rejection of science. Current Directions in Psychological Science, 25(4), 217-222. https://doi.org/10.1177/0963721416654436

Li, N., \& Su, L. Y.-F. (2018). Message framing and climate change communication: A meta- 
analytical review. Journal of Applied Communications, 102(3).

https://doi.org/10.4148/1051-0834.2189

*Ma, Y., Dixon, G., \& Hmielowski, J. D. (2019). Psychological reactance from reading basic facts on climate change: The role of prior views and political identification. Environmental Communication, 13(1), 71-86. https://doi.org/10.1080/17524032.2018.1548369

Maertens, R., Anseel, F., \& van der Linden, S. (2020). Combatting climate change misinformation: Evidence for longevity of inoculation and consensus messaging effects. Journal of Environmental Psychology, 70, 101455. https://doi.org/10.1016/j.jenvp.2020.101455

Magnusson, K. (2020). Interpreting Cohen's d effect size: An interactive visualization (Version 2.4.2) [Web App]. R Psychologist. https://rpsychologist.com/cohend/

Maki, A., Burns, R. J., Ha, L., \& Rothman, A. J. (2016). Paying people to protect the environment: A meta-analysis of financial incentive interventions to promote proenvironmental behaviors. Journal of Environmental Psychology, 47, 242-255. https://doi.org/10.1016/j.jenvp.2016.07.006

Maki, A., Carrico, A. R., Raimi, K. T., Truelove, H. B., Araujo, B., \& Yeung, K. L. (2019). Meta-analysis of pro-environmental behaviour spillover. Nature Sustainability, 2, 307-315. https://doi.org/10.1038/s41893-019-0263-9

Mann, M. E., Hassol, S. J., \& Toles, T. (2017, July 12). Doomsday scenarios are as harmful as climate change denial. The Washington Post. https://www.washingtonpost.com/opinions/doomsday-scenarios-are-as-harmful-as-climatechange-denial/2017/07/12/880ed002-6714-11e7-a1d7-9a32c91c6f40_story.html

*Manning, C., Mangas, H., Amel, E., Tang, H., Humes, L., Foo, R., Sidlova, V., \& Cargos, K. 
(2018). Psychological distance and response to human versus non-human victims of climate change. In W. Leal Filho, R. W. Marans, \& J. Callewaert (Eds.), Handbook of Sustainability and Social Science Research (pp. 143-161). Springer. https://doi.org/10.1007/978-3-31967122-2_8

Markowitz, E. M., \& Shariff, A. F. (2012). Climate change and moral judgement. Nature Climate Change, 2, 243-247. https://doi.org/10.1038/nclimate1378

McCright, A. M. (2010). The effects of gender on climate change knowledge and concern in the American public. Population and Environment, 32, 66-87. https://doi.org/10.1007/s11111010-0113-1

McCright, A. M., \& Dunlap, R. E. (2011). The politicization of climate change and polarization in the American public's views of global warming, 2001-2010. The Sociological Quarterly, 52(2), 155-194. https://doi.org/10.1111/j.1533-8525.2011.01198.x

McDonald, R. I., Chai, H. Y., \& Newell, B. R. (2015). Personal experience and the 'psychological distance' of climate change: An integrative review. Journal of Environmental Psychology, 44, 109-118. https://doi.org/10.1016/j.jenvp.2015.10.003

*Mildenberger, M., Lubell, M., \& Hummel, M. (2019). Personalized risk messaging can reduce climate concerns. Global Environmental Change, 55, 15-24. https://doi.org/10.1016/j.gloenvcha.2019.01.002

*Mildenberger, M., \& Tingley, D. (2019). Beliefs about climate beliefs: The importance of second-order opinions for climate politics. British Journal of Political Science, 49(4), 12791307. https://doi.org/10.1017/S0007123417000321

Mooney, C., Clement, S., \& Mufson, S. (2014, November 19). There's a global warming generation gap in the GOP, like on the issue of gay marriage. The Washington Post. 
https://www.washingtonpost.com/news/wonk/wp/2014/11/19/the-polls-are-clear-youngerrepublicans-support-action-on-climate-change/?noredirect $=$ on\&utm_term $=.74075$ e6ad005

Moser, S. C. (2010). Communicating climate change: History, challenges, process and future directions. Wiley Interdisciplinary Reviews: Climate Change, 1(1), 31-53. https://doi.org/10.1002/wcc.11

*Motta, M., Chapman, D., Stecula, D., \& Haglin, K. (2019). An experimental examination of measurement disparities in public climate change beliefs. Climatic Change, 154, 37-47. https://doi.org/10.1007/s10584-019-02406-9

*Myers, T. A., Maibach, E., Peters, E., \& Leiserowitz, A. (2015). Simple messages help set the record straight about scientific agreement on human-caused climate change: The results of two experiments. PLoS ONE, 10(3), e0120985. https://doi.org/10.1371/journal.pone.0120985

Nisa, C. F., Bélanger, J. J., Schumpe, B. M., \& Faller, D. G. (2019). Meta-analysis of randomised controlled trials testing behavioural interventions to promote household action on climate change. Nature Communications, 10, 4545. https://doi.org/10.1038/s41467-019$12457-2$

Osbaldiston, R., \& Schott, J. P. (2012). Environmental sustainability and behavioral science: Meta-analysis of proenvironmental behavior experiments. Environment and Behavior, 44(2), 257-299. https://doi.org/10.1177/0013916511402673

Palm, R., Lewis, G. B., \& Feng, B. (2017). What causes people to change their opinion about climate change? Annals of the American Association of Geographers, 107(4), 883-896. https://doi.org/10.1080/24694452.2016.1270193

Pearson, A. R., Schuldt, J. P., \& Romero-Canyas, R. (2016). Social climate science: A new vista 
for psychological science. Perspectives on Psychological Science, 11(5), 632-650. https://doi.org/10.1177/1745691616639726

Pechar, E., Bernauer, T., \& Mayer, F. (2018). Beyond political ideology: The impact of attitudes towards government and corporations on trust in science. Science Communication, 40(3), 291-313. https://doi.org/10.1177/1075547018763970

Pew. (2016, July 17). 2016 campaign: Strong interest, widespread dissatisfaction. Pew Research Center. https://www.people-press.org/2016/07/07/2016-campaign-strong-interestwidespread-dissatisfaction/

Pew. (2020, February 13). As economic concerns recede, environmental protection rises on the public's policy agenda. Pew Research Center. https://www.people-press.org/2020/02/13/aseconomic-concerns-recede-environmental-protection-rises-on-the-publics-policy-agenda/

*Porter, E., Wood, T. J., \& Bahador, B. (2019). Can presidential misinformation on climate change be corrected? Evidence from Internet and phone experiments. Research \& Politics, 6(3), 1-10. https://doi.org/10.1177/2053168019864784

Pustejovsky, J. E. (2020). clubSandwich: Cluster-robust (sandwich) variance estimators with small-sample corrections (R package version 0.4.1). https://cran.rproject.org/package $=$ clubSandwich

Pustejovsky, J. E., \& Rodgers, M. A. (2019). Testing for funnel plot asymmetry of standardized mean differences. Research Synthesis Methods, 10(1), 57-71. https://doi.org/10.1002/jrsm.1332

Ranney, M. A., \& Clark, D. (2016). Climate change conceptual change: Scientific information can transform attitudes. Topics in Cognitive Science, 8(1), 49-75. https://doi.org/10.1111/tops.12187 
Reser, J. P., \& Bradley, G. L. (2017). Fear appeals in climate change communication (Vol. 1). Oxford University Press. https://doi.org/10.1093/acrefore/9780190228620.013.386

Reynolds, J. P., Stautz, K., Pilling, M., van der Linden, S., \& Marteau, T. M. (2020).

Communicating the effectiveness and ineffectiveness of government policies and their impact on public support: A systematic review with meta-analysis. Royal Society Open Science, 7, 190522. https://doi.org/10.1098/rsos.190522

*Risen, J. L., \& Critcher, C. R. (2011). Visceral fit: While in a visceral state, associated states of the world seem more likely. Journal of Personality and Social Psychology, 100(5), 777793. https://doi.org/10.1037/a0022460

*Rode, J. B. (2019). Unpublished data. University of California, Irvine.

Rodgers, M. A., \& Pustejovsky, J. E. (2020). Evaluating meta-analytic methods to detect selective reporting in the presence of dependent effect sizes. Psychological Methods. Advance online publication. https://doi.org/10.1037/met0000300

*Schoenefeld, J. J., \& McCauley, M. R. (2016). Local is not always better: The impact of climate information on values, behavior and policy support. Journal of Environmental Studies and Sciences, 6, 724-732. https://doi.org/10.1007/s13412-015-0288-y

Schuldt, J. P., Konrath, S. H., \& Schwarz, N. (2011). “Global warming” or “climate change”?: Whether the planet is warming depends on question wording. Public Opinion Quarterly, 75(1), 115-124. https://doi.org/10.1093/poq/nfq073

*Schuldt, J. P., Pearson, A. R., Romero-Canyas, R., \& Larson-Konar, D. (2017). Brief exposure to Pope Francis heightens moral beliefs about climate change. Climatic Change, 141, 167177. https://doi.org/10.1007/s10584-016-1893-9

*Schuldt, J. P., \& Roh, S. (2014). Of accessibility and applicability: How heat-related cues affect 
belief in "global warming" versus "climate change." Social Cognition, 32(3), 217-238. https://doi.org/10.1521/soco.2014.32.3.217

Sears, D. O. (1986). College sophomores in the laboratory: Influences of a narrow data base on social psychology's view of human nature. Journal of Personality and Social Psychology, 51(3), 515-530. https://doi.org/10.1037/0022-3514.51.3.515

*Shin, F., \& Preston, J. L. (2019). Green as the gospel: The power of stewardship messages to improve climate change attitudes. Psychology of Religion and Spirituality. Advance online publication. https://doi.org/10.1037/rel0000249

Singh, A. S., Zwickle, A., Bruskotter, J. T., \& Wilson, R. (2017). The perceived psychological distance of climate change impacts and its influence on support for adaptation policy. Environmental Science \& Policy, 73, 93-99. https://doi.org/10.1016/j.envsci.2017.04.011

*Singh, S. P., \& Swanson, M. (2017). How issue frames shape beliefs about the importance of climate change policy across ideological and partisan groups. PLoS ONE, 12(7), e0181401. https://doi.org/10.1371/journal.pone.0181401

Skitka, L. J., Bauman, C. W., \& Sargis, E. G. (2005). Moral conviction: Another contributor to attitude strength or something more? Journal of Personality and Social Psychology, 88(6), 895-917. https://doi.org/10.1037/0022-3514.88.6.895

*Skurka, C., Niederdeppe, J., \& Nabi, R. (2019). Kimmel on climate: Disentangling the emotional ingredients of a satirical monologue. Science Communication, 41(4), 394-421. https://doi.org/10.1177/1075547019853837

*Skurka, C., Niederdeppe, J., Romero-Canyas, R., \& Acup, D. (2018). Pathways of influence in emotional appeals: Benefits and tradeoffs of using fear or humor to promote climate change-related intentions and risk perceptions. Journal of Communication, 68(1), 169-193. 
https://doi.org/10.1093/joc/jqx008

Smith, N., \& Leiserowitz, A. (2014). The role of emotion in global warming policy support and opposition. Risk Analysis, 34(5), 937-948. https://doi.org/10.1111/risa.12140

Soutter, A. R. B., \& Mõttus, R. (2020). "Global warming" versus "climate change": A replication on the association between political self-identification, question wording, and environmental beliefs. Journal of Environmental Psychology, 69, 101413. https://doi.org/10.1016/j.jenvp.2020.101413

*Sparks, A. (2017). Reducing distance to increase action: How psychological proximity drives activism [Doctoral dissertation, University of California, Santa Barbara]. https://escholarship.org/uc/item/7nq534jf

Spence, A., \& Pidgeon, N. (2010). Framing and communicating climate change: The effects of distance and outcome frame manipulations. Global Environmental Change, 20(4), 656-667. https://doi.org/10.1016/j.gloenvcha.2010.07.002

Stang, A., Poole, C., \& Bender, R. (2010). Common problems related to the use of number needed to treat. Journal of Clinical Epidemiology, 63(8), 820-825. https://doi.org/10.1016/j.jclinepi.2009.08.006

*Stroik, P., Chakraborty, D., Ge, W., Boulter, J., \& Jamelske, E. (2019). Effect of reciprocity on public opinion of international climate treaties: Experimental evidence from the US and China. Climate Policy, 19(8), 959-973. https://doi.org/10.1080/14693062.2019.1617666

Stroud, N. J. (2010). Polarization and partisan selective exposure. Journal of Communication, 60(3), 556-576. https://doi.org/10.1111/j.1460-2466.2010.01497.x

Suldovsky, B. (2017). The information deficit model and climate change communication. In Oxford Research Encyclopedia of Climate Science. Oxford University Press. 
https://doi.org/10.1093/acrefore/9780190228620.013.301

Taber, C. S., \& Lodge, M. (2006). Motivated skepticism in the evaluation of political beliefs. American Journal of Political Science, 50(3), 755-769. https://doi.org/10.1111/j.15405907.2006.00214.x

Tannenbaum, M. B., Hepler, J., Zimmerman, R. S., Saul, L., Jacobs, S., Wilson, K., \& Albarracín, D. (2015). Appealing to fear: A meta-analysis of fear appeal effectiveness and theories. Psychological Bulletin, 141(6), 1178-1204. https://doi.org/10.1037/a0039729

Tanner-Smith, E. E., \& Tipton, E. (2014). Robust variance estimation with dependent effect sizes: Practical considerations including a software tutorial in Stata and SPSS. Research Synthesis Methods, 5(1), 13-30. https://doi.org/10.1002/jrsm.1091

Tanner-Smith, E. E., Tipton, E., \& Polanin, J. R. (2016). Handling complex meta-analytic data structures using robust variance estimates: A tutorial in R. Journal of Developmental and Life-Course Criminology, 2, 85-112. https://doi.org/10.1007/s40865-016-0026-5

*Tesler, M. (2018). Elite domination of public doubts about climate change (not evolution). Political Communication, 35(2), 306-326. https://doi.org/10.1080/10584609.2017.1380092

Tipton, E. (2015). Small sample adjustments for robust variance estimation with metaregression. Psychological Methods, 20(3), 375-393. https://doi.org/10.1037/met0000011

Tipton, E., \& Pustejovsky, J. E. (2015). Small-sample adjustments for tests of moderators and model fit using robust variance estimation in meta-regression. Journal of Educational and Behavioral Statistics, 40(6), 604-634. https://doi.org/10.3102/1076998615606099

Trope, Y., \& Liberman, N. (2010). Construal-level theory of psychological distance. Psychological Review, 117(2), 440-463. https://doi.org/10.1037/a0018963

*Truelove, H. B., Yeung, K. L., Carrico, A. R., Gillis, A. J., \& Raimi, K. T. (2016). From plastic 
bottle recycling to policy support: An experimental test of pro-environmental spillover. Journal of Environmental Psychology, 46, 55-66.

https://doi.org/10.1016/j.jenvp.2016.03.004

*Tvinnereim, E., Lachapelle, E., \& Borick, C. (2016). Is support for international climate action conditional on perceptions of reciprocity? Evidence from survey experiments in Canada, the US, Norway, and Sweden. COSMOS, 12(01), 43-55. https://doi.org/10.1142/S0219607716500038

*van der Linden, S. L., Leiserowitz, A. A., Feinberg, G. D., \& Maibach, E. W. (2014). How to communicate the scientific consensus on climate change: Plain facts, pie charts or metaphors? Climatic Change, 126, 255-262. https://doi.org/10.1007/s10584-014-1190-4 van der Linden, S. L., Leiserowitz, A. A., Feinberg, G. D., \& Maibach, E. W. (2015). The scientific consensus on climate change as a gateway belief: Experimental evidence. PLoS ONE, 10(2), e0118489. https://doi.org/10.1371/journal.pone.0118489

van der Linden, S., Leiserowitz, A., \& Maibach, E. (2017). Gateway illusion or cultural cognition confusion? Journal of Science Communication, 16(05). https://doi.org/10.22323/2.16050204

van der Linden, S., Leiserowitz, A., \& Maibach, E. (2018). Perceptions of scientific consensus predict later beliefs about the reality of climate change using cross-lagged panel analysis: A response to Kerr and Wilson (2018). Journal of Environmental Psychology, 60, 110-111. https://doi.org/10.1016/j.jenvp.2018.10.002

*van der Linden, S., Leiserowitz, A., \& Maibach, E. (2019). The gateway belief model: A largescale replication. Journal of Environmental Psychology, 62, 49-58.

https://doi.org/10.1016/j.jenvp.2019.01.009 
*van der Linden, S., Leiserowitz, A., Rosenthal, S., \& Maibach, E. (2017). Inoculating the public against misinformation about climate change. Global Challenges, l(2), 1600008. https://doi.org/10.1002/gch2.201600008

van der Linden, S., Maibach, E., \& Leiserowitz, A. (2019). Exposure to scientific consensus does not cause psychological reactance. Environmental Communication, 1-8. https://doi.org/10.1080/17524032.2019.1617763

Wallace-Wells, D. (2017, July). The uninhabitable Earth. New York Magazine. https://nymag.com/intelligencer/2017/07/climate-change-earth-too-hot-for-humans.html Washburn, A. N., \& Skitka, L. J. (2018). Science denial across the political divide: Liberals and conservatives are similarly motivated to deny attitude-inconsistent science. Social Psychological and Personality Science, 9(8), 972-980. https://doi.org/10.1177/1948550617731500

*Watkins, H. M., \& Goodwin, G. P. (2020). Reflecting on sacrifices made by past generations increases a sense of obligation towards future generations. Personality and Social Psychology Bulletin, 46(7), 995-1012. https://doi.org/10.1177/0146167219883610

Weber, E. U. (2016). What shapes perceptions of climate change? New research since 2010. Wiley Interdisciplinary Reviews: Climate Change, 7(1), 125-134. https://doi.org/10.1002/wcc.377

Wilson, D. B. (n.d.). Practical meta-analysis effect size calculator [Online calculator].

Witte, K., \& Allen, M. (2000). A Meta-Analysis of fear appeals: Implications for effective public health campaigns. Health Education \& Behavior, 27(5), 591-615. https://doi.org/10.1177/109019810002700506

*Wolske, K. S., Raimi, K. T., Campbell-Arvai, V., \& Hart, P. S. (2019). Public support for 
carbon dioxide removal strategies: The role of tampering with nature perceptions. Climatic Change, 152, 345-361. https://doi.org/10.1007/s10584-019-02375-z

Wolsko, C. (2017). Expanding the range of environmental values: Political orientation, moral foundations, and the common ingroup. Journal of Environmental Psychology, 51, 284-294. https://doi.org/10.1016/j.jenvp.2017.04.005

Wolsko, C., Ariceaga, H., \& Seiden, J. (2016). Red, white, and blue enough to be green: Effects of moral framing on climate change attitudes and conservation behaviors. Journal of Experimental Social Psychology, 65, 7-19. https://doi.org/10.1016/j.jesp.2016.02.005

*Wong-Parodi, G., \& Fischhoff, B. (2015). The impacts of political cues and practical information on climate change decisions. Environmental Research Letters, 10(3), 034004. https://doi.org/10.1088/1748-9326/10/3/034004

*Zaval, L., Markowitz, E. M., \& Weber, E. U. (2015). How will I be remembered? Conserving the environment for the sake of one's legacy. Psychological Science, 26(2), 231-236. https://doi.org/10.1177/0956797614561266

*Zhou, J. (2016). Boomerangs versus javelins: How polarization constrains communication on climate change. Environmental Politics, 25(5), 788-811. https://doi.org/10.1080/09644016.2016.1166602 


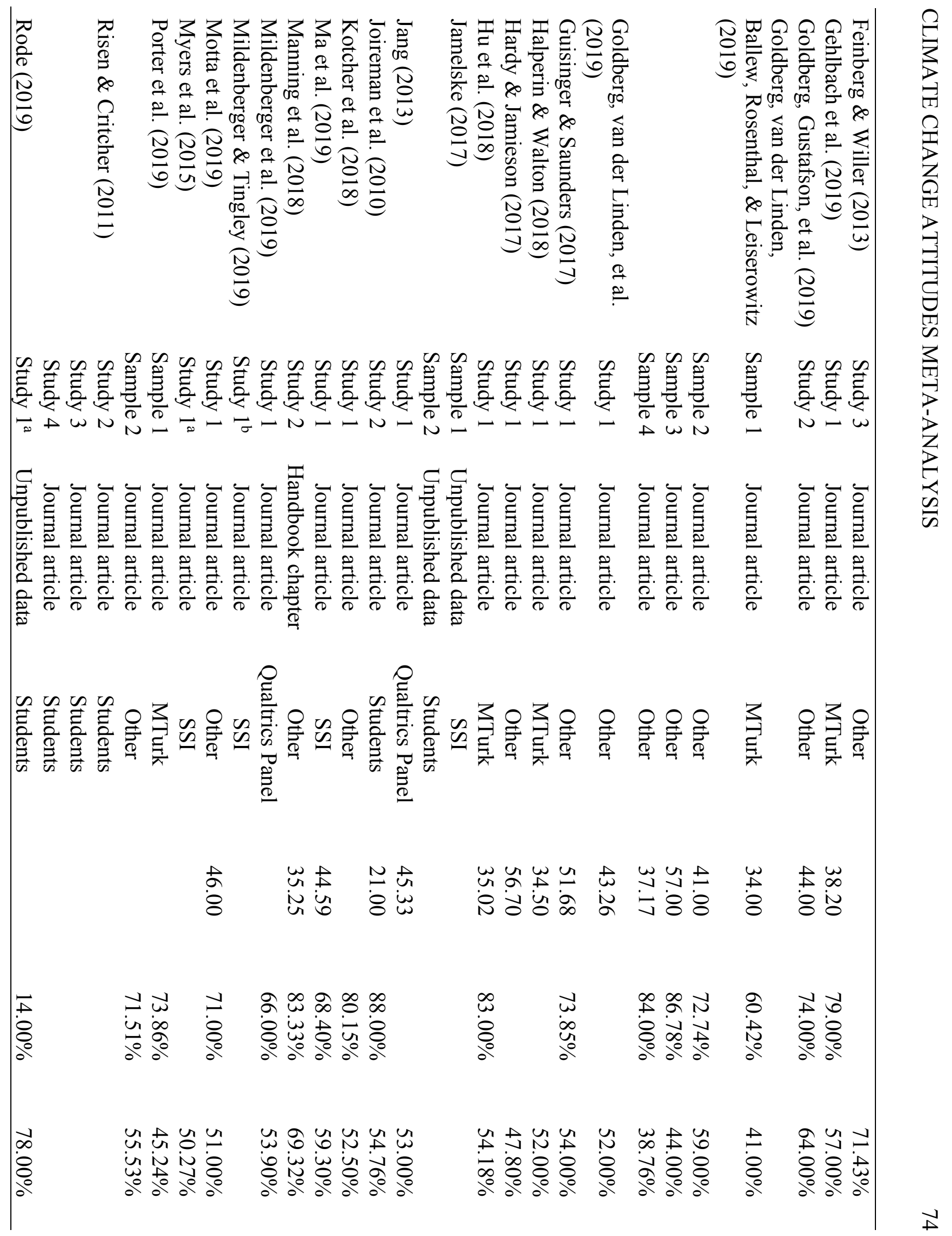




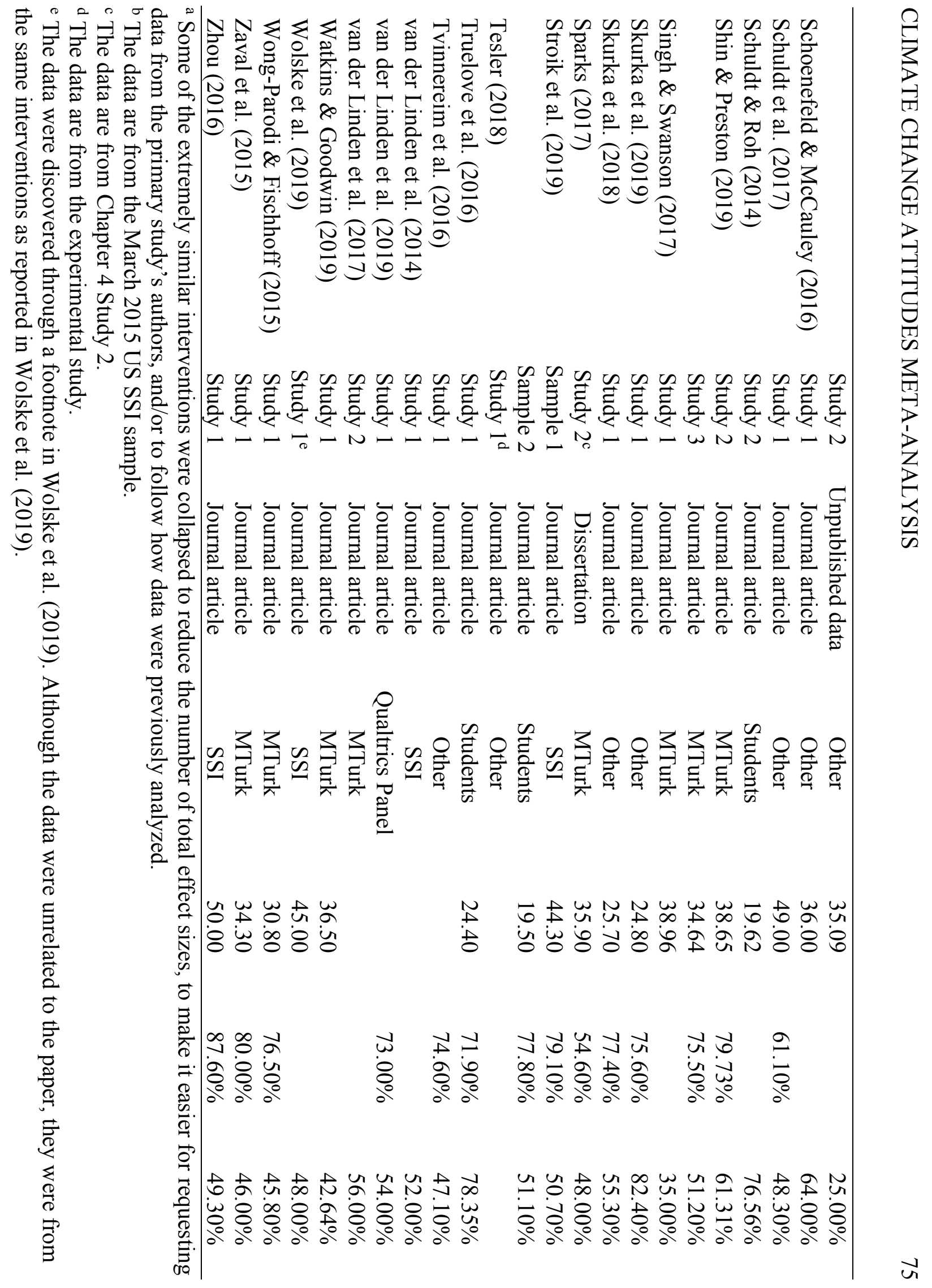

